\section{Action Plan for Stroke in Europe 2018-2030}

\author{
Bo Norrving', Jon Barrick ${ }^{2}$, Antoni Davalos ${ }^{3}$, Martin Dichgans ${ }^{4}$, \\ Charlotte Cordonnier ${ }^{5}$, Alla Guekht ${ }^{6}$, Kursad Kutluk ${ }^{7}$, \\ Robert Mikulik ${ }^{8}$, Joanna Wardlaw', Edo Richard ${ }^{10}$, \\ Darius Nabavi '", Carlos Molina ${ }^{12}$, Philip M Bath ${ }^{13}$, \\ Katharina Stibrant Sunnerhagen ${ }^{14}$, Anthony Rudd ${ }^{15}$, \\ Avril Drummond ${ }^{16}$, Anna Planas ${ }^{17}$ and Valeria Caso ${ }^{18}$; \\ on behalf of the Action Plan for Stroke in Europe \\ Working Group*
}

European Stroke Journal 2018, Vol. 3(4) 309-336 (C) European Stroke Organisation 2018

\section{(c) (1) \$}

Article reuse guidelines: sagepub.com/journals-permissions DOI: $10.1177 / 2396987318808719$ journals.sagepub.com/home/eso

\section{@SAGE}

\begin{abstract}
Two previous pan-European consensus meetings, the 1995 and 2006 Helsingborg meetings, were convened to review the scientific evidence and the state of current services to identify priorities for research and development and to set targets for the development of stroke care for the decade to follow. Adhering to the same format, the European Stroke Organisation (ESO) prepared a European Stroke Action Plan (ESAP) for the years 2018 to 2030, in cooperation with the Stroke Alliance for Europe (SAFE). The ESAP included seven domains: primary prevention, organisation of stroke services, management of acute stroke, secondary prevention, rehabilitation, evaluation of stroke outcome and quality assessment and life after stroke. Research priorities for translational stroke research were also identified. Documents were prepared by a working group and were open to public comments. The final document was prepared after a workshop in Munich on 2I-23 March 2018. Four overarching targets for 2030 were identified: (I) to reduce the absolute number of strokes in Europe by 10\%, (2) to treat $90 \%$ or more of all patients with stroke in Europe in a dedicated stroke unit as the first level of care, (3) to have national plans for stroke encompassing the entire chain of care, (4) to fully implement national strategies for multisector public health interventions. Overall, 30 targets and 72 research priorities were identified for the seven domains. The ESAP provides a basic road map and sets targets for the implementation of evidence-based preventive actions and stroke services to 2030.
\end{abstract}

'Department of Clinical Sciences Lund, Neurology, Skåne University Hospital, Lund University, Lund, Sweden

${ }^{2}$ Stroke Alliance for Europe, London, UK

${ }^{3}$ Department of Neurosciences, Hospital Universitari Germans Trias i Pujol, Universitat Autònoma de Barcelona, Barcelona, Spain ${ }^{4}$ Institute for Stroke and Dementia Research, University Hospital, Ludwig-Maximilians University, Munich, and Munich Cluster of Systems Neurology (SyNergy), Munich, Germany

${ }^{5}$ Department of Neurology, CHU Lille, Lille, France

${ }^{6}$ Clinical Center for Neuropsychiatry, Russian National Research Medical University, Moscow, Russia

${ }^{7}$ Department of Neurology, Stroke Unit, University of Dokuz Eylul, Izmir, Turkey

${ }^{8}$ International Clinical Research Center and Neurology Department, St Anne's University Hospital Brno and Masaryk University Brno, Czech Republic

${ }^{9}$ Centre for Clinical Neurosciences, Edinburgh Imaging and UK Dementia Research Institute, University of Edinburgh, Edinburgh, UK

${ }^{10}$ Department of Neurology, Radboud University Medical Centre, Nijmegen, and Department of Neurology, Academic Medical Centre, Amsterdam, the Netherlands

\footnotetext{
"Department of Neurology with Stroke Unit, Vivantes Hospital Neukölln, Berlin, Germany

${ }^{12}$ Stroke Unit, Department of Neurology, Hospital Vall d'Hebron Barcelona, Spain

${ }^{13}$ Stroke Trials Unit, Division of Clinical Neuroscience, University of Nottingham, Nottingham, UK

${ }^{14}$ Institute of Neuroscience and Physiology, Sahlgrenska University Hospital, Gothenburg, Sweden

${ }^{15}$ Guy's and St Thomas' NHS Foundation Trust, Stroke NHS England and Royal College of Physicians, London, UK

${ }^{16}$ School of Health Sciences, University of Nottingham, Nottingham, UK

${ }^{17}$ Institut d'Investigacions Biomèdiques de Barcelona (IIBB), Institut d'Investigacions Biomèdiques August Pi i Sunyer (IDIBAPS),

Barcelona, Spain

${ }^{18}$ Stroke Unit, Department of Medicine and Cardiovascular Medicine, University of Perugia, Perugia, Italy

*See Appendix I for the full list of the working group members.
}

\section{Corresponding author:}

Bo Norrving, Department of Clinical Sciences, Neurology, Department of Neurology, Skåne University Hospital, S-22I 86 Lund, Sweden.

Email: bo.norrving@med.lu.se 


\section{Keywords}

Stroke, epidemiology, prevention, treatment, stroke services, strategic planning, treaties, quality assurance, Europe

Date received: 26 August 2018; accepted: I October 2018

\section{Introduction}

Stroke remains one of the leading causes of death and disability in Europe, and projections show that with a 'business as usual' approach, the burden of stroke will not decrease in the next decade or beyond. An important contributing factor to this is that the number of older persons in Europe is rising, with a projected increase of $35 \%$ between 2017 and $2050{ }^{1}$ Fortunately, there is compelling evidence that stroke is highly preventable, treatable and manageable, and the potential exists to drastically reduce the burden of stroke and its long-term consequences. However, this requires the joint actions of ministries of health, other governmental bodies, scientific and stroke support organisations, healthcare professionals, clinical and preclinical researchers and the pharmaceutical and device industries.

To this end, two previous pan-European consensus meetings, the 1995 and 2006 Helsingborg meetings, 2,3 were convened to review the scientific evidence and the state of current services and to set targets for the development of stroke care for the decade to follow. The European Stroke Organisation (ESO) has prepared a European Stroke Action Plan (ESAP) for the years 2018 to 2030, in cooperation with the Stroke Alliance for Europe (SAFE). The ESAP adheres to the format of the Helsingborg Declarations, presenting a review of the 'state of the art', the state of current services, research and development priorities and targets for a series of domains in stroke care (organisation of stroke services, management of acute stroke, prevention, rehabilitation, evaluation of stroke outcome and quality assessment). The ESAP includes two additional domains, on primary prevention and life after stroke, along with research and development priorities for translational stroke research. ESAP 2018-2030 complements the WHO Global Action Plan on noncommunicable diseases (NCDs) 2013-2020, the WHO-Europe NCD Action Plan and the UN Sustainable Development Goals for 2015 to 2030.

\section{Overarching targets for 2030}

For each domain of the 2018 to 2030 ESAP, specific targets are being set, as detailed in the following sections. Beyond these targets, four overarching targets for 2030 have been identified:

1. to reduce the absolute number of strokes in Europe by $10 \%$

2. to treat $90 \%$ or more of all patients with stroke in Europe in a dedicated stroke unit as the first level of care

3. to have national plans for stroke encompassing the entire chain of care from primary prevention to life after stroke.

4. to fully implement national strategies for multisector public health interventions to promote and facilitate a healthy lifestyle, and reduce environmental (including air pollution), socioeconomic and educational factors that increase the risk of stroke.

\section{Methods}

The work was led by a steering committee. The documents were prepared by working groups for each of the seven domains, with patient organisation representatives in each domain. Research priorities were identified by two persons for each domain. One working group prepared research priorities for translational stroke research. The draft documents were open to public comments during a one-month period. The final document was prepared after a workshop in Munich on 21-23 March 2018 that was streamed live.

\section{Primary prevention}

\section{State of the art}

Primary prevention of stroke is part of both primary healthcare and public health. Stroke prevention, which involves both pharmacological and nonpharmacological interventions, targets many of the same risk factors as those involved in other cardiovascular diseases (CVDs) and other NCDs. Thus, primary prevention of stroke has two integral components, which can be implemented at different levels. First, measures to promote a healthy lifestyle, such as smoking cessation and reducing alcohol intake, can be implemented at the individual patient level. Second, reductions in air pollution and improvements in 
socioeconomic and educational status require action at governmental and societal level.

Risk factor modification. Ten modifiable risk factors may account for the vast majority of the population attributable risk of stroke, across age groups, genders and ethnicity. These risk factors are: hypertension, smoking, dyslipidaemia, unhealthy diet, physical inactivity, obesity, diabetes mellitus, cardiac disease, excessive alcohol intake and psychosocial factors. ${ }^{4}$ There is overwhelming evidence that the treatment of cardiovascular risk factors reduces the risk of stroke, but the target levels in primary prevention are less stringent than those in secondary prevention and depend on comorbidities such as diabetes mellitus and active smoking; these targets may differ between men and women. ${ }^{5}$ There is good evidence to support risk factor modification as primary prevention in individuals aged 40-75 years, but the optimal target levels for the management of hypertension and dyslipidaemia in persons outside this age range are less well established. ${ }^{5,6}$

Hypertension, diabetes mellitus, dyslipidaemia, smoking, unhealthy diet, obesity, physical inactivity and atrial fibrillation (AF) are the strongest and most common modifiable risk factors for stroke, ${ }^{7,8}$ and most guidelines include these risk factors. Based on an extensive literature, most primary prevention guidelines recommend the use of risk tables, which take age and comorbidities into account. In a European setting, the Systematic COronary Risk Evaluation (SCORE) tables, accessible through the European Society of Cardiology website, are appropriate, since they are derived from a mixed European population. An adapted version for older people, SCORE-OP, is available. ${ }^{9}$

Self-management, with or without the use of technology such as eHealth and mobile health (mHealth) initiatives, may offer the possibility of delivering primary prevention in a range of healthcare settings, including geographically remote areas in Europe. However, the expected impact on risk factor improvement is modest at best, and sustainability of effects is a major challenge. ${ }^{10} \mathrm{~A}$ consistent and constructive collaboration between healthcare professionals in primary, secondary and tertiary care is likely to be essential for sustainable primary prevention.

Primary prevention in public health. Primary prevention can be delivered at the individual, community and population levels. Public health interventions targeting highly prevalent risk factors that do not require pharmacological intervention, including discouraging smoking and encouraging a healthy lifestyle, should be implemented at several levels; such interventions may include legislative changes, media campaigns, labelling of food and educational and preventive measures in schools, workplaces and the community. Although there is only limited high-quality evidence for a direct effect on the incidence of stroke and CVD, initiatives such as building cycle lanes, guiding people to stairs, serving healthy food in public places, smoking bans, decreasing the amount of salt and sugar in processed food and soft drinks, health education and public health campaigns to increase awareness of modifiable stroke risk factors are sensible public health interventions and should be pursued.

From a population perspective, it is not sufficient to target only those individuals at high risk of CVD. The majority of strokes will develop in persons with low or intermediate risk, ${ }^{11}$ and hence broad prevention strategies targeting the wider population are indicated, particularly considering the high prevalence of some of the strongest risk factors for stroke. A combination of opportunistic screening of those at increased risk (based, for example, on demographic characteristics) and systematic screening for risk factors is probably most appropriate. ${ }^{5}$ In particular, raising awareness of hypertension as a risk factor for stroke could increase early detection rates and lead to earlier initiation of treatment. Many risk factors are more prevalent among people with low socioeconomic status, and extra effort to reach these people with preventive strategies is warranted. ${ }^{12}$ In all situations, the potential benefits and risks - such as over-medicalisation - should be carefully evaluated.

\section{State of current services}

There are major differences among European countries, indirectly reflecting the state of local services, in terms of risk factor prevalence and control. ${ }^{7}$ Moreover, awareness of the modifiable risk factors for stroke can vary markedly between populations. ${ }^{8}$ The organisation of primary prevention services differs greatly between European countries. Some countries, such as the Netherlands and the United Kingdom, have a strong primary care system, and extensive guidelines that include the primary prevention of CVD are in place, whereas in other countries, it is less clear who is principally responsible for primary prevention. There are currently no generally accepted European guidelines as to which populations should be screened, for which risk factors and at which age. A national NCD action plan is a prerequisite for successful primary prevention strategies that are feasible and realistic for each country, but not all European countries have such a plan. ${ }^{12}$

Blood pressure management. The prevalence of hypertension in people above the age of 18 years ranges from 20 to $40 \%$ in Europe. ${ }^{8}$ Of those on medical treatment, 
only a minority sustainably reach their target blood pressure. ${ }^{13}$

Smoking. A steady decline in smoking since 1990 has occurred among men and women in most European countries. However, $10-28 \%$ of the adult populations in European countries still smoke. ${ }^{14,15}$

Obesity, diet and physical activity. The prevalence of overweight and obesity has increased dramatically over the last 25 years. National programmes to reduce salt consumption have been successful in many European countries, with high evidence of a beneficial effect on cardiovascular mortality in some countries. Furthermore, 6 out of 10 European citizens do not reach the WHO-recommended $150 \mathrm{~min}$ of moderate intensity physical activity per week. ${ }^{7}$

Dyslipidaemia. The number of people with untreated dyslipidaemia has decreased over the last 25 years, but adherence to treatment with cholesterol-lowering drugs is still poor, with less than half of patients being sustainably adherent to their medication. ${ }^{16}$

Diabetes mellitus. Diabetes care can be organised in outpatient clinics or in primary care, coordinated by specialised nurses. The percentage of patients with adequate diabetes control can be estimated based on the glycated haemoglobin $\left(\mathrm{HbA}_{1 \mathrm{C}}\right)$ level and varies between European countries, reflecting different levels of service.

Atrial fibrillation. AF-related stroke is increasing in incidence and prevalence: this may be related to better detection but is at least partly the result of the increasing incidence of AF with age and the ageing population in most countries. In people with AF with an appropriate $\mathrm{CHA}_{2} \mathrm{DS}_{2} \mathrm{VASc}$ score, the beneficial effect of anticoagulation is evident, but the value of wider screening for AF and the clinical significance of short episodes of paroxysmal AF are currently under debate, particularly if it concerns primary prevention. The balance between benefits and harms of long-term anticoagulation as primary prevention is questionable in some AF patients, particularly those with very short episodes of AF or a low $\mathrm{CHA}_{2} \mathrm{DS}_{2}$ VASc score. ${ }^{17}$

Because stroke and CVDs share many common risk factors, research initiatives in primary prevention of stroke should not be undertaken in isolation. Close collaboration with primary prevention initiatives from cardiology, primary healthcare and public health is strongly recommended and should be covered by a regional or national NCD action plan. Such initiatives should be aligned with WHO initiatives such as the
Action Plan for NCD, and updated as necessary when these are revised.

\section{Research and development priorities}

1. Which factors are responsible for major health disparities in Europe, including risk factor prevalence and control, and access and adherence to primary prevention? What are the effects of low socioeconomic status and other social factors?

2. Can the current risk prediction models be improved by extending the current 10 -year risk prediction to 20-year or life-time risk for those at younger age ( $<40$ years), and five-year or life-time risk for those over 75 years?

3. Can further individualisation of primary prevention strategies, for example, by considering multiple comorbidities, poly-pharmacy, geographic and ethnic differences and polygenetic risk profiles, enhance effectiveness?

4. Can public awareness of the potential for primary prevention of stroke be improved by personalised and public health education about modifiable risk factors?

5. What are the benefits and harms of screening for stroke risk factors in different populations, using different approaches such as systematic and opportunistic screening?

6. Can adherence to primary prevention interventions be improved by using eHealth or mHealth approaches to encourage self-management, using drug combinations (for example, in 'polypill' formulations), and combining individualised approaches with public health interventions?

\section{Targets for 2030}

1. Achieving universal access in Europe to primary preventive treatments based on improved and more personalised risk prediction.

2. Full implementation of national strategies for multisectorial public health interventions promoting and facilitating a healthy lifestyle, and reducing environmental, socioeconomic and educational factors that increase the risk of stroke.

3. Making available evidence-based screening and treatment programmes for stroke risk factors in all European countries.

4. Having blood pressure detected and controlled in $80 \%$ of persons with hypertension.

As with the research and development priorities, close collaboration and alignment with cardiovascular disease prevention targets are essential to meet these proposed targets. 


\section{Organisation of stroke services}

\section{State of the art}

The organisation of stroke services is fundamental to quality care across the spectrum of care that progresses from prevention via acute therapy to long-term care. Definitions of the different facilities for stroke care included into this document are provided in Table 1. The focus of this section is on the organisation of acute stroke treatment services, since other elements will be presented in the sections dealing with specific domains. Although organised services exist in most European countries, there is large variability in the practical application of treatment guidelines and adherence to quality indicators. ${ }^{19}$ Awareness programmes have been shown to have an impact on the number of acute stroke calls, but this effect is not sustained in the long term. ${ }^{20}$ National or regional quality registries have been implemented in several countries to monitor key performance indicators, and programmes to certify stroke units and stroke centres have been established in others. ${ }^{21}$ Research activities, covering both experimental and clinical studies, and funding have significantly increased during the last decades. ${ }^{22}$

Appropriate training of emergency medical services (EMS) personnel and dispatchers increases the number of patients arriving early at hospital, and several countries have established clear transportation routes to the closest suitable hospital. ${ }^{23}$ Pre-hospital identification of patients with acute stroke by use of validated tools and scales has been recognised as being important for prompt treatment: the Face Arm Speech Test (FAST) is the easiest and the most commonly used, but all prehospital scales demonstrate suboptimal specificity, even with adequate training. ${ }^{24,25}$ The development of stroke teams (including all disciplines required for acute stroke management including radiology) has been shown to lead to shorter delays and more rapid management, particularly when the team is pre-notified of the imminent arrival of the patient. ${ }^{26,27}$ The concept of delivering personnel and equipment to the patient via mobile stroke units seems to be effective, but this option is not widely available. ${ }^{28}$ The same is true for helicopter transportation, which may be useful in certain settings. ${ }^{29}$

Admission to centralised, rather than noncentralised, facilities for acute hospital care increases the likelihood of receiving thrombolytic treatment, which has a direct impact on stroke outcome. ${ }^{30}$ Hospitals with greater use of thrombolysis achieve statistically and clinically significantly shorter delays in administering tissue plasminogen activator (tPA) after arrival in hospital. ${ }^{31}$ This may be also true for mechanical thrombectomy, but data on this are limited at
Table I. Definitions of selected terms used in the European Stroke Action Plan.

Stroke unit: A dedicated geographically clearly defined area or ward in a hospital, where stroke patients are admitted and cared for by a multi-professional team (medical, nursing and therapy staff) who have specialist knowledge of cerebral function, training and skills in stroke care with well-defined individual tasks, regular interaction with other disciplines and stroke leadership. This team coordinates care through regular, multidisciplinary meetings. ${ }^{18}$

Stroke centre: A hospital infrastructure and related processes of care that provide the full pathway of stroke unit care. A stroke centre is the coordinating body of the entire chain of care. This covers pre-hospital care, ongoing rehabilitation and secondary prevention and access to neurosurgical and vascular intervention. A stroke unit is the most important component of a stroke centre. The Stroke Centre provides stroke unit services for the population of its own catchment area and serves as a referral centre for peripheral hospitals with stroke units in case their patients need services which are not available locally. ${ }^{18}$

Comprehensive stroke unit: A dedicated area where acute stroke management is combined with early mobilisation and rehabilitation and secondary prevention, according to the needs of the patient.

Early supported discharge: Early supported discharge is designed to enable the accelerated discharge of stroke patients to their home, providing specialist rehabilitation and social support in a home setting rather than an acute hospital ward. The early supported discharge team comprises a variety of specialist therapists, social and support workers. The team visits patients in their home setting, enabling patients to undergo rehab in a familiar home setting, and thus increasing patient flow and bed availability within the acute hospital.

Registry: A system for collecting process and outcome data at regional or national level that achieves near universal coverage.

present. Treatment in dedicated stroke units significantly reduces the risk of disability, institutional care and death by $20 \%$, irrespective of stroke severity and age. ${ }^{32}$ All hospitals should ensure that patients with suspected stroke have rapid access to brain imaging, including vascular imaging at all times, since immediate brain imaging is the most cost-effective approach in stroke. ${ }^{33}$ Early supported discharge (ESD) in a multidisciplinary setting is beneficial for patients with mild to moderate stroke and is a likely cause of the significant reduction in length of stay in stroke units in these patients. $^{34,35}$

In remote regions without specialist stroke expertise, telemedicine in community hospitals may help to provide evidence-based acute stroke treatment, including thrombolysis. ${ }^{36}$ Certification and sentinel stroke audit programmes support the delivery of high-quality organised care: it has been shown that high performance in audited process indicators reduces mortality by up to $25 \%{ }^{37}$ This emphasises the need for 
continuing quality improvement processes, particularly in view of the known differences in care quality related to time and day of admission. ${ }^{38}$ Auditing measures lead to a better quality of stroke care, and more sustained stroke care performance over time. ${ }^{39}$

\section{State of current services}

Political awareness of the burden of stroke has increased in recent years, but still varies significantly across Europe. Although significant progress has been made, there is still large heterogeneity in the organisation of stroke care in Europe, as shown by a recent ESO survey in 44 countries. ${ }^{40}$ Hence, reliable and precise information about the structure and organisation of stroke care, and the implementation of stroke management pathways, is still lacking in many countries. In most, but not all, European countries, health services and political structures are linked to a national stroke society, and these linkages help to coordinate stroke services and foster quality improvements of stroke care. Most countries have an EMS system with regional organisation and written protocols for acute stroke. However, many do not have obligatory transport routes to the closest suitable hospital or procedures for pre-hospital notification of hyper-acute stroke. Although stroke symptoms and the importance of immediate action have repeatedly been communicated to the public, awareness is still unsatisfactory among the general population.

The crucial impact of time in acute stroke has constantly been stressed, but fewer than $10 \%$ of stroke patients reach the hospital within $60 \mathrm{~min}$ of symptom onset. In many countries, the symptom-to-hospital delay has not decreased in recent years. Several countries have built a nationwide network of hospitals with stroke units or stroke centres following written protocols. However, only limited information on the definitions of stroke units and comprehensive stroke centres in the various countries is available. Moreover, only a minority of countries have established a certification system with well-defined quality criteria, or a monitored system for benchmarking delivery of care, that is evaluated at regular intervals. Among the options for acute stroke care, mechanical thrombectomy is the latest and the most challenging component, and there is currently huge variation in the delivery of this treatment across Europe. ${ }^{40}$

Only a few countries have established a continuous quality improvement system with a predefined set of criteria that are regularly measured and compared with benchmarks. The reimbursement structures for stroke care are still highly variable between European countries, leading to gaps in care quality in some countries. The challenges for acute stroke care differ markedly between countries, and between different regions (e.g. cities, rural areas), but only a few countries monitor the delivery of stroke care at a patient level to ensure a defined standard of service.

\section{Research and development priorities}

1. Can we identify the most relevant barriers to the implementation of evidence-based stroke care? What is the role of economical aspects?

2. What is the health-economic impact of strokes and the return of investment in stroke care?

3. Which are the most cost-effective concepts to improve the organisation of stroke care in countries with limited resources?

4. Which are the minimum educational criteria for stroke experts (physicians, nurses or therapists)?

5. What are the optimum numbers and ratios of stroke centres and stroke units for municipal and rural areas?

6. What is the role of telemedicine systems for acute stroke, rehabilitation and long-term care?

7. What is the appropriate structure to manage childhood stroke?

8. How can regional networks of EMS, stroke units and rehabilitation centres be developed most effectively?

9. How can evidence-based media campaigns be organised in order to promote public awareness and knowledge of stroke signs and the importance of immediate action?

10. What elements are needed to enable more effective participation in decision making among patients and relatives?

\section{Targets for 2030}

1. Establishing a medical society and stroke support organisation in each country, which collaborates closely with the responsible body in developing, implementing and auditing the national stroke plan.

2. Guiding national stroke care by evidence-based pathways that cover the entire chain of care. These pathways are understood by the public and may be adapted to meet regional circumstances to ensure equal access to stroke care irrespective of patient characteristics, region and time of hospitalisation.

3. Managing and delivering stroke care by competent personnel and teams, and creating plans for effective recruitment and training as part of a national stroke plan.

4. All stroke units and other stroke services undergo regular certifications or equivalent auditing processes for quality improvement. 


\section{Management of acute stroke}

\section{State of the art}

Acute stroke is a medical emergency. The benefit of recanalisation therapies in patients with acute ischaemic stroke is strongly time-dependent, with earlier intervention achieving better outcomes. ${ }^{41}$ Stroke care systems should therefore minimise the time to assessment and initiation of treatment, before brain injury becomes irreversible. ${ }^{42,43}$ All patients with ischaemic stroke or intracranial haemorrhage (ICH) benefit from specialist attention and organised care within a designated stroke unit (number needed to treat (NNT) to prevent poor outcome, 18) including rapid diagnostic imaging, probably due in part to the prevention or early treatment of complications such as infections and fever. ${ }^{32}$ Randomised trials testing strategies to prevent complications are ongoing. ${ }^{32,44}$

For ischaemic stroke, aspirin - started within two days of the onset of symptoms - has a small benefit (NNT 79) but is suitable for a large number of patients. Although unproven, the benefit of early treatment with clopidogrel is probably comparable. In selected patients with acute ischaemic stroke or TIA, dual antiplatelet therapy in the first weeks confers additional benefit. ${ }^{45}$ Selected interventions, including intravenous thrombolysis, endovascular thrombectomy (EVT) and hemicraniectomy, offer greater benefit but are applicable to fewer patients than antiplatelet therapy. Timely restoration of blood flow through intravenous thrombolysis (IVT, NNT 5-9) or EVT (NNT 3) is the first priority. The earlier the treatment with IVT is initiated, the greater the benefit, irrespective of age or stroke severity. ${ }^{42}$ In patients with acute ischaemic stroke caused by occlusion of a proximal intracranial artery of the anterior circulation, EVT strongly increases the chance of a good outcome and, like alteplase, improves global functional outcomes. The initial evidence of benefit with EVT was largely limited to patients in whom treatment could be started within $6 \mathrm{~h}$ of symptom onset, but recent evidence suggests that, in highly selected patients with acute ischaemic stroke and considerable residual mismatch between perfusion deficit and infarct volume, EVT can offer benefit when used up to $24 \mathrm{~h}$ of symptom onset. In recent trials, EVT was associated with a $19 \%$ absolute reduction in the risk of a poor outcome, but nevertheless $29-67 \%$ of the patients randomised to the intervention arm were dead or dependent at three months. ${ }^{46-50}$

The wake up study reported that in selected patients with acute stroke with an unknown time of onset, intravenous alteplase guided by a mismatch between diffusion-weighted imaging (DWI) and fluid-attenuated inversion recovery (FLAIR) in the region of ischemia is beneficial. ${ }^{51}$

For patients aged 60 years or younger with large, space-occupying infarctions, surgical decompression involving hemicraniectomy and duraplasty strongly reduces the risk of death or dependency (NNT 2-4). ${ }^{52}$ The benefit in older patients is questionable because surgical decompression in the elderly reduces the risk of death at the cost of a large increase in the risk of long-term dependency in activities of daily living (ADL). ${ }^{53}$ For patients with $\mathrm{ICH}$, care on a stroke unit is the only treatment that has been demonstrated to be beneficial. Early intensive blood pressure lowering may improve outcome, although the NNT is relatively large. ${ }^{54}$ To date, the use of haemostatic agents to prevent early haematoma growth has not been proven effective. Surgical treatment may be life-saving in some patients, but a surgical intervention policy has not been shown to improve outcome in patients with $\mathrm{ICH}$ in general. $^{55,56}$

In patients with subarachnoid haemorrhage $(\mathrm{SAH})$ caused by a rupture of an intracranial aneurysm, the primary goal is the prevention and treatment of complications such as rebleeding, delayed cerebral ischaemia and hydrocephalus. The risk of rebleeding can be reduced by occlusion of the aneurysm with endovascular or microneurosurgical techniques; coiling is preferred in cases where both treatment options appear equally feasible. Oral nimodipine reduces the risk of delayed cerebral ischaemia and increases the chance of a favourable outcome. ${ }^{57,58}$ Early, short-term, treatment with tranexamic acid to reduce the risk of recurrent SAH before the aneurysm is secured, is currently under study.

Many in-hospital deaths of stroke patients occur after a decision to withhold or withdraw lifesustaining treatments. Observational studies have demonstrated a strong relation between treatment restrictions, such as do-not-resuscitate orders and an increased risk of in-hospital mortality. It remains uncertain whether this relation is causal, and whether refraining from do-not-resuscitate orders would result in better outcomes. ${ }^{59}$

\section{State of current services}

A recent survey of access to, and delivery rates of, acute stroke unit care, IVT and EVT in 44 European countries found major inequalities in acute stroke treatment between and within countries. ${ }^{40}$ Overall, there were 1.5 stroke units per 1000 annual incident ischaemic strokes, but individual country-level data indicate that both access to and delivery of acute stroke care are insufficient or lacking in many countries. Furthermore, regional differences were reported within 28 countries. 
Fewer than $20 \%$ of patients with acute ischaemic stroke had access to treatment with IVT, and the overall rate of IVT in incident ischaemic stroke was 7.3\%, with considerable inter- and intra-country variability. EVT was performed in only 0.4 centres per 1000 annual incident ischaemic strokes, and only $1.9 \%$ of all acute ischaemic stroke patients were estimated to have received this treatment. Moreover, 28 countries lacked full EVT coverage. ${ }^{40}$

The mainly Western European SITS-MOST registry has reported a median onset-to-treatment time of $140 \mathrm{~min}$, compared with $150 \mathrm{~min}$ reported by the Eastern European SITS-EAST registry. A median door-to-needle (DNT) time of $70 \mathrm{~min}$ has been reported in international European registries; continuous improvement of processes can limit median DNT times to just $20 \mathrm{~min}$ in experienced and highvolume centres.

Guidelines for the management of ICH generally recommend early blood pressure control in patients with acute ICH. Unfortunately, European data on the use of antihypertensive treatment in this setting, and the degree of blood pressure control achieved, are scarce.

\section{Research and development priorities}

1. How can disparities in access to stroke unit care across European countries be diminished?

2. Which reperfusion options should be used, based on patient-, service- and cost-specific factors?

3. How can the speed, safety and effectiveness of reperfusion approaches (drugs or devices) be optimised in Europe?

4. Which pharmacological or other strategies will reduce the extent of irreversible brain damage in ischaemic stroke patients before recanalisation therapies are started?

5. Which strategies will improve outcomes in ischaemic stroke patients who are not eligible for reperfusion therapies, or who do not recover after recanalisation?

6. Which treatment strategies will improve outcomes in patients with ICH: haemostatic and surgical approaches, prevention of secondary injury, or intensive and tailored blood pressure management?

7. Which treatment strategies will further improve outcomes in patients with SAH by reducing brain injury?

\section{Targets for 2030}

1. Treating $90 \%$ or more of all patients with stroke in Europe in a stroke unit as the first level of care.
2. Guaranteeing access to recanalisation therapies to $95 \%$ of eligible patients across Europe.

3. Decreasing median onset-to-needle times to $<120 \mathrm{~min}$ for intravenous thrombolysis and onsetto-reperfusion times to $<200 \mathrm{~min}$ for endovascular treatment.

4. Achieving IVT rates above $15 \%$, and EVT rates above $5 \%$, in all European countries.

5. Decreasing first-month case-fatality rates to $<25 \%$ for $\mathrm{ICH}$, and increasing the rate of good functional outcomes to $>50 \%$.

6. Decreasing first-month case-fatality rates to $<25 \%$ for $\mathrm{SAH}$, and increasing the rate of good functional outcomes to $>50 \%$.

\section{Secondary prevention}

\section{State of the art}

In the Helsingborg Declaration of $2006,{ }^{3}$ the goals for 2015 were to reduce stroke mortality by at least $20 \%$, and for all patients with TIA or stroke to receive appropriate secondary preventive measures. Within Europe, all countries agree that stroke risk factors, most importantly hypertension and smoking, should be reduced in their populations, but implementation of secondary prevention measures varies considerably between countries, with declining success rates from west to east.

Secondary prevention encompasses the reduction of further stroke and TIA, any other vascular disease, and other complications including cognitive decline and dementia, mood disturbances or anxiety, fatigue and poor quality of life. Secondary prevention applies to almost all patients with stroke or TIA and can reduce stroke recurrence by $80 \%$. Investigation and treatment must commence in hospital (stroke unit or stroke clinic) and continue throughout life in the community. Applicable ESO and national guidelines are regularly updated, and most investigations and interventions are available to healthcare systems across Europe. The routine use of these guidelines will ensure equity of access and equality of care across Europe.

Investigations. Investigations of incident stroke, and its cause(s) and risk factors, should follow guideline-based local protocols including rapid and appropriate brain and vascular imaging (Table 2); recurrent strokes should be re-investigated since the mechanism may be different from that of the original stroke. If magnetic resonance imaging (MRI) is performed, it should include T2, FLAIR, DWI and blood-sensitive sequences. If there is no obvious cause of stroke, identifying paroxysmal $\mathrm{AF}$ and patent foramen ovale (PFO) become important. 
Table 2. Recommendations for the investigation of incident or recurrent stroke.

\begin{tabular}{|c|c|c|}
\hline Stroke type & Aim & Investigation \\
\hline \multirow[t]{4}{*}{ All } & $\begin{array}{l}\text { Ischaemic vs. } \\
\text { haemorrhagic }\end{array}$ & $\begin{array}{l}\mathrm{CT} \pm \mathrm{CTA} \text {, or } \mathrm{MRI} \pm \mathrm{MRA} \text {, scanning immediately on admission } \\
\text { to hospital }\end{array}$ \\
\hline & Vital measures & Blood pressure, weight/body mass index \\
\hline & Blood tests & $\begin{array}{l}\text { Lipids, glucose, HbAlc, coagulation, markers for vasculitis and con- } \\
\text { nective tissue disorders }\end{array}$ \\
\hline & Severe high BP & For secondary causes of hypertension \\
\hline \multirow[t]{5}{*}{ Ischaemic/TIA } & Large artery stroke & Carotid ultrasound (extracranial) \\
\hline & & $\mathrm{CT}$ and $\mathrm{CTA}$, or MRI and MRA \\
\hline & Atrial fibrillation & ECG \\
\hline & & Prolonged arrhythmia recording \\
\hline & Embolic stroke & $\begin{array}{l}\text { Echocardiography and bubble contrast transcranial Doppler if per- } \\
\text { formed locally }\end{array}$ \\
\hline $\begin{array}{l}\text { Intracerebral } \\
\text { haemorrhage }\end{array}$ & & $\begin{array}{l}\text { CTA or MRA; digital subtraction angiography if appropriate. Interval } \\
\text { blood-sensitive MRI }\end{array}$ \\
\hline $\begin{array}{l}\text { Subarachnoid } \\
\text { haemorrhage }\end{array}$ & & $\begin{array}{l}\text { CT and CTA, lumbar puncture; digital subtraction angiography if } \\
\text { appropriate. Delayed CTA or MRA }\end{array}$ \\
\hline
\end{tabular}

Note: Investigations may not be relevant or appropriate in all patients, e.g. those with dependent dementia or other causes of a reduced life-expectancy.

BP: blood pressure; CT: computed tomography; CTA: computed tomography angiography; ECG: electrocardiogram; HbA Ic: glycated haemoglobin; MRA: magnetic resonance angiography; MRI: magnetic resonance imaging.

The identification of risk factors has two major cornerstones: identifying 'lifestyle' risk factors that would benefit from a personalised approach and 'medical' risk factors for which management guidelines exist. Modifiable risk factors such as poor diet, alcohol and tobacco use, drug addiction, obesity, high blood pressure and cholesterol, AF, diabetes mellitus and sleep apnoea, must be addressed in every patient (Table 3). Non-modifiable factors, such as age, sex, race or ethnicity and family history, modulate the importance of modifiable factors: national stroke registers should record the presence of these factors, and interventions used to prevent or treat modifiable risk factors.

The minimum standard of care for secondary prevention consists of lifestyle advice and lowering elevated blood pressure; in patients with ischaemic events, antithrombotic therapy, a statin and carotid endarterectomy (if appropriate) should be added. Patient and carer education about prevention strategies ${ }^{60}$ should be delivered at every opportunity by all healthcare professionals working in stroke care. Interventions should be started in hospital, with targets that are defined by a multidisciplinary panel and reviewed on a regular basis. The effect of treatment on blood pressure and lipids should be assessed at follow-up.

Following the diagnosis of ischaemic stroke or TIA, the aetiological cause (large artery disease, cardioembolism, small vessel disease (SVD), stroke of other determined aetiology and stroke of undetermined aetiology) should be identified. This approach allows treatment with appropriate secondary preventative strategies
(Table 3); this summary of interventions will be updated in future ESO and other guidelines. A similar approach should be taken for the diagnosis of $\mathrm{ICH}$, which may be lobar (typically due to cerebral amyloid angiopathy) or deep (typically related to hypertension) and may also be related to rupture of an aneurysm or arteriovenous malformation, or other aetiologies (Table 3).

Surgical or radiological procedures such as carotid endarterectomy and stenting, and closure of PFO and the atrial appendage, are highly operator dependent. Success rates should be monitored and are dependent on proper training and mentoring, and on performing an adequate number of procedures each year.

Patients require long-term follow-up to monitor adherence to therapy and the development of cerebrovascular events. Typically, this is done in the community, but the use of home-based point-of-care devices and wearables may improve follow-up data collection and diagnosis of subsequent events.

Most patients benefit from investigation and preventative interventions after a stroke or TIA, and advanced age is not a contraindication. However, patients with significant frailty, dementia or dependency might be spared some prevention strategies by taking account of their wishes and those of their family. Patients with stroke or TIA often have co-existing cardiac, renal or peripheral arterial disease, and this needs investigation and treatment. Depression and anxiety can be detrimental to lifestyle and adherence and are risk factors for stroke and dementia. 
Table 3. Effective secondary prevention interventions and their targets according to type of stroke.

\begin{tabular}{|c|c|}
\hline Stroke type & Intervention \\
\hline All & $\begin{array}{l}\text { - Primary prevention measures are applicable to secondary prevention } \\
\text { - Government campaigns, e.g. promoting healthy lifestyle, reducing air pollution } \\
\text { - Stop smoking ( } \pm \text { nicotine replacement) and added salt, moderate alcohol intake and weight, }{ }^{61} \text { and } \\
\text { increase exercise }{ }^{62} \text { and consumption of a stroke-protective diet including fruit and vegetables } \\
\text { - Treat high blood pressure with angiotensin-converting enzyme inhibitors or angiotensin receptor } \\
\text { antagonists, calcium channel blockers and/or thiazide-like diuretics; }{ }^{63} \text { usually needs } \geq 2 \text { agents to } \\
\text { reach target of }<130 / 80 \mathrm{mmHg} \text { (if tolerated). Patients needing } \geq 3 \text { drugs to reach target should } \\
\text { be investigated for secondary causes of hypertension } \\
\text { - Glucose lowering in diabetes mellitus. Pioglitazone may provide prevention benefits beyond } \\
\text { - } \text { glucose control } \\
\text { mone-replacement therapy may increase the frequency and severity of stroke }\end{array}$ \\
\hline $\begin{array}{l}\text { Ischaemic stroke/transient } \\
\text { ischaemic attack }\end{array}$ & $\begin{array}{l}\text { - Antiplatelet for non-cardioembolic stroke (e.g. AF). }{ }^{64} \text { Dual therapy based on aspirin and clopi- } \\
\text { dogrel for three weeks in acute minor stroke and TIA-, then mono-therapy, or aspirin and } \\
\text { dipyridamole, is relevant for lacunar infarction }\end{array}$ \\
\hline Large artery disease & $\begin{array}{l}\text { - Lipid lowering with a statin, ideally at maximum dose. }{ }^{65} \text { Fibrates, ezetimibe or PCSK9 inhibitors } \\
\text { may be prescribed if statins cannot be tolerated or give insufficient response } \\
\text { - CEA or carotid stenting for symptomatic severe ipsilateral carotid stenosis (NASCET score } \\
\geq 70 \% \text { ) or in most high-risk patients with moderate stenosis ( } 50-69 \% \text { ), as soon as the patient is } \\
\text { stable and within two weeks. }{ }^{66} \text { If CEA is not possible, or in younger patients, extracranial carotid } \\
\text { artery stenting is an alternative for high-risk carotid stenosis }\end{array}$ \\
\hline Cardioembolic stroke & $\begin{array}{l}\text { - In atrial fibrillation, NOAC rather than with warfarin or other vitamin } \mathrm{K} \text { antagonists. }{ }^{67} \text { Although } \\
\text { currently on-patent and therefore more expensive, NOACs have no prothrombotic properties } \\
\text { on initiation, provide more consistent anticoagulation, reduce the risk of intracranial haemor- } \\
\text { rhage, do not need monitoring and have less interactions with food and other drugs } \\
\text { - In patients with likely embolism (no lacunar or large artery features), cardiac echocardiography } \\
\text { (or bubble transcranial Doppler) should be performed. Device closure of PFO }{ }^{68} \text { with a moderate } \\
\text { to large shunt or an atrial aneurysm reduces recurrent events in patients }<60 \text { years }\end{array}$ \\
\hline Lacunar stroke & - Optimise control of blood pressure, blood glucose and lipids and mono-antiplatelet therapy \\
\hline Other determined aetiology & $\begin{array}{l}\text { - These include cervical artery dissection, cerebral venous thrombosis, recreational drugs and } \\
\text { genetic mutations and require specific investigation and treatment (in addition to treatment as } \\
\text { defined in 'All' above) }\end{array}$ \\
\hline Intracerebral haemorrhage & - Lower raised blood pressure (as above) \\
\hline Subarachnoid haemorrhage & $\begin{array}{l}\text { - Stop smoking, moderate alcohol intake and lower raised blood pressure (as above). Non-invasive } \\
\text { screening for first-degree family }\end{array}$ \\
\hline
\end{tabular}

Note: Interventions may not be relevant or appropriate in all patients, related to adverse events, concurrent conditions (e.g. dependent dementia). AF: atrial fibrillation; CEA: carotid endarterectomy; NASCET: North American Symptomatic Carotid Endarterectomy Trial; NOAC: non-vitamin K oral anticoagulant; PCSK9: proprotein convertase subtilisin/kexin type 9; PFO: patent foramen ovale.

\section{State of current services}

Although there is wide variation in the provision of secondary prevention across Europe, accurate country-specific data are not available for most interventions, and published data are usually 5-10 years old. This indicates an urgent need for more accurate monitoring and reporting of secondary prevention (Appendix 2). Available data suggest that more than $60 \%$ of patients presenting with a stroke are hypertensive; although $80-90 \%$ of these are treated, fewer than $40 \%$ will have their blood pressure adequately controlled. Reasons for inadequate blood pressure control include a lack of monitoring, under-treatment and low adherence rates. Similarly, although statin prescription rates on discharge from hospital are high, long-term adherence rates are low. Furthermore, many patients with AF still do not receive oral anticoagulation.

\section{Research and development priorities}

For all patients

- Do technological solutions improve compliance?

- Do biomarkers identify patients at high of stroke recurrence and improve secondary prevention?

- Can identification of non-responders to antithrombotic therapy improve secondary prevention?

- Can prevention be enhanced in patients taking recommended prophylaxis?

- Which preventive measures are beneficial in the very old? 
- What preventive therapies are effective in sporadic small vessel (lacunar) stroke and monogenic cerebrovascular disorders, such as CADASIL (Cerebral Autosomal Dominant Arteriopathy with Subcortical Infarcts and Leukoencephalopathy) and Anderson Fabry disease?

\section{Healthcare delivery}

- Should patients with a minor stroke or TIA be rapidly assessed in a fast-track one-stop TIA clinic or in the stroke unit following admission?

- Should all patients be followed up long-term, e.g. at one year?

\section{Lifestyle and risk factors}

- What is the optimal blood pressure target for each type and subtype of stroke?

- Does treatment of sleep apnoea reduce stroke recurrence?

- Which nutritional interventions physical activities reduce stroke recurrence?

\section{Large artery atherosclerosis}

- Does best medical therapy obviate the need for carotid intervention in asymptomatic patients?

- Are new antithrombotic strategies required?

- Will advanced stenting procedures further reduce recurrence?

- Will new lipid-lowering drugs further reduce stroke recurrence?

- Should patients be investigated for asymptomatic atherosclerosis?

- What is the optimal treatment for intracranial stenosis causing recurrent events?

\section{Cardioembolism}

- Which patients benefit from PFO closure?

- What is the optimal treatment strategy in patients with AF and a high risk of ischaemic and haemorrhagic stroke?

- What is the optimal time to start oral anticoagulation after AF-associated stroke?

- How should patients with AF and atherosclerosis be treated?

\section{SVD}

- How can SVD-related cerebral lesions and their progression be prevented?

- Should the presence or burden of SVD lesions influence secondary prevention decisions?

- Which preventive therapies are effective?

\section{$\mathrm{ICH}$}

- Which patients with AF on oral anticoagulants can be restarted with oral anticoagulants following $\mathrm{ICH}$ ?

- When should statins be (re-)started after ICH in patients with ischaemic vascular disease?

SAH

- Understand the mechanisms of aneurysm formation and rupture in order to prevent recurrent $\mathrm{SAH}$.

\section{Targets for 2030}

1. Including secondary prevention in national stroke plans with follow-up in primary/community care.

2. Ensuring that at least $90 \%$ of the stroke population is seen by a stroke specialist and have access to secondary prevention management (investigation and treatment).

3. Ensure access to key investigational modalities: CT (or MR) scanning, carotid ultrasound, ECG, 24-h ECG, echocardiography (transthoracic and transoesophageal), blood tests (lipids, glucose, $\mathrm{HbA}_{1 \mathrm{c}}$, coagulation, erythrocyte sedimentation rate, C-reactive protein and autoantibodies).

4. Ensuring access to key preventative strategies: lifestyle advice, antihypertensives, lipid-lowering agents, antiplatelets, anticoagulants, oral hypoglycaemic agents and insulin, carotid endarterectomy and PFO closure.

\section{Rehabilitation}

\section{State of the art}

In the Helsingborg Declaration of $2006,{ }^{3}$ the goal for 2015 was that all stroke patients in Europe would have access to a continuum of care from acute management to appropriate rehabilitation, delivered in dedicated stroke units.

Among adults, stroke is the most common cause of new disability leading to more than one impairment that could affect daily activities (Table 4). Rehabilitation has been defined by the WHO as 'a set of measures that assist individuals, who experience or are likely to experience disability, to achieve and maintain optimal functioning in interaction with their environments'. ${ }^{69}$ It is instrumental in enabling people with functional limitations to remain in or return to their home or community, live independently and participate in education, work and civic life. The United Nations Convention on the Rights of Persons with Disabilities (UNCRPD) includes the right to rehabilitation, ${ }^{70}$ but the 2017 SAFE report on Stroke in Europe ${ }^{8}$ has clearly shown that access to rehabilitation varies across Europe. In the same year, WHO made a call for 
Table 4. Distribution of neurological deficits after stroke. ${ }^{a}$

\begin{tabular}{llll}
\hline Affected area & Frequency & Consequences & Treatment \\
\hline Motor function & $50-85 \%$ & $\begin{array}{c}\text { Impaired balance, transfer ability, walking and } \\
\text { reduced upper extremity function }\end{array}$ & $\begin{array}{c}\text { Task-specific repetition training seems } \\
\text { most beneficial }\end{array}$ \\
Cognition & $\approx \mathrm{I} / 2$ & $\begin{array}{c}\text { Memory problems, reduced attention, execu- } \\
\text { tive dysfunction and spatial neglect } \\
\text { May affect the person's ability to manage } \\
\text { daily life. }\end{array}$ & $\begin{array}{c}\text { No clear evidence of beneficial inter- } \\
\text { ventions. So far, often-compensatory } \\
\text { strategies seem to work best }\end{array}$ \\
Communication & $\approx \mathrm{I} / 3$ & $\begin{array}{c}\text { Aphia, ranging from occasional word-finding } \\
\text { difficulties to having no effective means of } \\
\text { verbal communication }\end{array}$ & $\begin{array}{c}\text { Information to patient and family in the } \\
\text { acute setting }\end{array}$ \\
\hline
\end{tabular}

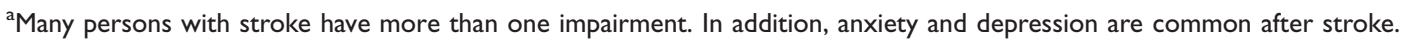

action, 'Rehabilitation 2030', to address the significant rehabilitation needs across the world and the substantial apathy towards these needs shown by many governments. $^{71}$

Care in hospital - stroke units and early rehabilitation. Acute stroke care, skilled nursing care and specialist rehabilitation are all core aspects of a comprehensive stroke unit, and treatment in such facilities has been shown to reduce mortality and disability. ${ }^{31}$ Rehabilitation includes occupational, physical and speech and language therapy, with input from psychologists and social workers as necessary. It should involve a multidisciplinary approach from stroke physicians in a comprehensive stroke team, and an ordered plan for discharge from hospital with documented responsibility for continuing rehabilitation needs in the community.

Patients and carers need information about stroke, rehabilitation and planned discharge and follow-up. ${ }^{72}$ This information improves knowledge, patient satisfaction and reduces depression. How this information is best delivered remains unclear, but active involvement of both patients and family members seems to be beneficial.

Transition from hospital to home - early supported discharge and community rehabilitation. $\mathrm{ESD}^{34,35}$ is an innovative approach to rehabilitation, in which services are provided at home by a mobile rehabilitation team, and should be seen as a part of the stroke treatment pathway. The target audience is mainly patients with mild or moderate stroke symptoms, which includes about $30 \%$ of the stroke population in most settings. Studies show that access to ESD services reduces length of hospital stay by a mean of six days and reduces the odds of being dead or dependent after stroke by $20 \%$. Patients with moderate to severe stroke should still have access to stroke rehabilitation, either during a prolonged stay in hospital or with adequate rehabilitation to address their needs in the community.
As recovery continues and stamina increases, the intensity of training may increase. For patients with aphasia, this can mean high-intensity training over a prolonged period, which has proven effective in terms of improved functional communication, reading, writing and expressive language. ${ }^{73}$

There is evidence that continued ADL training in the home setting after discharge has a beneficial effect for up to one year after stroke. ${ }^{74}$ Different modes of rehabilitation delivery during the first year, such as domiciliary, day-case hospital care and home-based care have been shown to improve independence in personal ADL.

Stroke survivors are often physically deconditioned, with muscle weakness in both the affected and unaffected sides, and impairments in cardiorespiratory fitness. Physical fitness training after stroke reduces disability, improves walking ability and may improve other stroke-related deficits such as cognition, mood and fatigue. ${ }^{75,76}$ Access to green areas and walking space may facilitate physical activity. ${ }^{77-79}$

Later rehabilitation and reintegration. Whether rehabilitation provided for more than one year after a stroke can improve recovery remains unclear. People who are recovering from a stroke often have persistent disabilities after one year or more. A Cochrane review has found that there are few studies in this area, and hence data are inconclusive, although there appears to be a tendency towards improved recovery with rehabilitation. ${ }^{76}$ However, targeted training, such as balance training, gait training with different devices, and upper extremity training more than one year after stroke seems to have a positive effect. ${ }^{75,80-82}$ Language training in specific groups or with computer programs has also been reported to be beneficial. This can be performed in peer-support patient organisation groups, or in other forms of group training. ${ }^{83}$ For younger persons, returning to work raises issues of identity and quality of life, as well as a means of living; hence, access to vocational rehabilitation is 
important for patients in whom a return to work seems possible. ${ }^{84}$

It is important to recognise that improvement can continue for a long time after stroke, and that the patient's needs will vary over time. Therefore, it is never too late for rehabilitation.

\section{State of current services}

There is large variability in access to rehabilitation between and within countries in Europe. These differences probably reflect international differences in the organisation of stroke services, strategic approaches to stroke and available resources. Comprehensive stroke units are still lacking in many countries, and the slow rehabilitation documented in stroke unit trials is almost absent. Patients with moderate to severe stroke benefit even more from stroke unit care. If this is not possible, the stroke rehabilitation unit provides an evidencebased alternative for continuing in-patient treatment.

There is a need to increase the number and capacity of comprehensive stroke units, in order to ensure that all patients have equitable access. There is also a shortage of rehabilitation and nursing staff with expertise in stroke and an understanding of rehabilitation. Rehabilitation in a comprehensive stroke unit should be available at all times.

There is a shortage of ESD services in all European countries, and in some areas, this is not offered as part of the stroke pathway. Similarly, physical fitness training programmes are not common in Europe but are now being developed in Italy and the UK.

\section{Research and development priorities}

- How can we efficiently implement long-term rehabilitation strategies, including measures such as ESD?

- How can we deliver more cost-effective long-term rehabilitation?

- Do self-management programmes improve longterm outcomes of stroke rehabilitation?

- How can rehabilitation be implemented with evidence-based results on timing, level and type of intervention?

- How can we improve participation and integration into society among stroke survivors?

\section{Management of neurological deficits}

- Can post-stroke fatigue be more effectively managed?

- Can post-stroke visual problems be more effectively managed?

- Can post-stroke language problems be more effectively managed?
- Can post-stroke cognitive impairment be more effectively managed?

- Can depression or anxiety be more effectively managed?

- Can post-stroke upper limb problems be more effectively managed?

- Can rehabilitation in an enriched environment improve outcome?

\section{Targets for 2030}

1. Guaranteeing that at least $90 \%$ of the population have access to early rehabilitation within the stroke unit.

2. Providing ESD to at least $20 \%$ of stroke survivors in all countries.

3. Offering physical fitness programmes to all stroke survivors living in the community.

4. Providing a documented plan for community rehabilitation and self-management support for all stroke patients with residual difficulties on discharge from hospital.

5. Ensuring that all stroke patients and carers have a review of the rehabilitation and other needs at three to six months after stroke and annually thereafter.

\section{Evaluation of outcomes and quality improvement}

\section{State of the art}

Background. There are huge variations in the quality of care for stroke patients in Europe, both between and within countries. This is partly due to differential access to resources, but often it is due to less easily explained differences in the organisation and delivery of care. The goal set for 2015 in the Helsingborg Declaration of 2006 was that all countries should establish a system for the routine collection of data needed to evaluate the quality of stroke management including patient safety issues. ${ }^{3}$ This has been only partially achieved, but there is evidence of benefit in those countries that have successfully implemented quality improvement programmes. The Action Plan for Stroke in Europe 2018-2030 aims to raise the overall standards of care that patients can expect, in accordance with international evidence-based practice and to enable weaker services to improve.

Guidelines. Comprehensive guidelines need to be developed and used to set standards for clinical services. While international guidelines such as the ESO stroke guidelines can form the basis for national guidelines, these need to be adapted for use within different health 
systems. The guidelines should form the basis for developing audit standards.

Measuring quality (audit). Clear standards and assessment of the quality of stroke services are essential if quality improvement is to be achieved. This information is needed to allow individual clinicians to monitor and modify their practice, for stroke services (hospital and community), for funders of healthcare to ensure that funded services are being adequately delivered, and for healthcare planners at national and international level. Additionally, it is important that the results of quality monitoring, with appropriate interpretation, are made available to patients and the public, to provide assurance that high-quality care is being delivered. If valid and reliable comparisons are to be made between services nationally and internationally, it is essential that definitions and terminology are standardised across countries. One of the reasons for apparent differences in incidence and outcomes after stroke is that different countries collect and report data in different ways. This is particularly evident with the definition of TIA, but there are also variations in the way that International Classification of Diseases (ICD) codes are used to classify stroke.

To fully describe a service, data are needed on the structure of stroke services (facilities, staffing, etc.), on the processes of care (door to needle times, intensity of rehabilitation provided, time to get to a stroke unit, etc.) and outcomes (mortality, physical, cognitive and psychological disability, frequency of complications such as pneumonia, etc.). The measures used should address aspects of care for the whole patient pathway, including acute care, rehabilitation, post-hospital care and secondary prevention. It is important both to record the care provided and the patients' experience of care and patient-reported outcomes. Registry data can be a powerful research tool to confirm that randomised controlled trial (RCT) evidence is transferable to routine services and can be used to address questions that could never be tested in RCTs.

Supporting improvement. Different audit models have been used across Europe, ranging from 'snapshot audit' where a consecutive cohort of patients have their care quality measured for a short period to audits that attempt to collect data on all stroke patients. However, collecting and reporting data alone is not sufficient to produce quality improvement: support is needed to enable change. This may involve local clinical leadership, managerial support, external help through peer review and sometimes the provision of additional resources. Sometimes, a major service reconfiguration may be required.

\section{State of current services}

Health service structures and payment systems often determine the pathways of care. The organisation of stroke services varies considerably around Europe, with stroke patients being managed within general neurology services, by full-time stroke specialists, or within general medical services. Specialist care is common for the hyperacute phase of treatment, but rehabilitation is more often managed separately within generic rehabilitation facilities. Community care is even more diverse, with few countries extending specialist care to domiciliary services. Few countries or regions have appointed a national clinical lead for stroke, with the exception of England, Scotland and Ireland. National guidelines for stroke management have been produced in many countries, including the UK, Sweden, the Republic of Ireland, Spain, France and Italy. In addition, some European Guidelines have been developed to cover specific aspects of stroke care, and others are in development. ${ }^{85}$

There are few international comparisons of care that are based on high-quality data. The 2017 SAFE report ${ }^{8}$ is the most up-to-date picture of stroke care in Europe but does not address quality of care. However, based partly on the Global Burden of Disease studies, it does show large variations in case fatality rates, which range from $3 \%$ in Denmark to $18 \%$ in Latvia. ${ }^{8}$ Research studies, some funded by the EU, have compared stroke care in different countries, but these are usually performed at individual hospital level rather than nationally.

Comprehensive stroke quality registers (with participation from all hospitals treating acute stroke patients) are available in some countries and regions, including Sweden, the UK, Germany, Catalonia, the Republic of Ireland, the Netherlands, Norway and Finland. A quality register is operating in Eastern Europe (RES-Q), which is rapidly recruiting participating centres. However, only Sweden and the UK attempt to provide data for patients after leaving hospital, and few countries make the quality data available to the general public. Regional, rather than national, initiatives for quality improvement are in place in some countries, such as Spain and Italy, and this can result in significant within-country variations in care quality. Quality indicators for stroke have been published in France and Turkey, and a consensus paper on core standards for measuring quality has been published. ${ }^{86}$

\section{Research and development priorities}

1. What definitions should be used across Europe for the recording and reporting of data on stroke and TIA? 
2. What are the most effective and efficient systems for the collection of data on the quality of stroke care across Europe?

3. How can data on the quality of care be used to compare process and outcomes of care, taking into account variations in case-mix, and what is the minimum dataset that is needed?

4. What are the systems needed to allow international comparisons of the clinical and cost-effectiveness of care, and reporting of within-country variations and variations by other factors such as geographical region (urban versus rural), and variations over time?

5. What are the most effective quality improvement tools that will enable learning from examples of best practice?

\section{Targets for 2030}

1. Defining a Common European Framework of Reference for Stroke Care Quality that includes: (a) development or update of European guidelines for management of acute stroke care, longer term rehabilitation and prevention; (b) definition of a common dataset covering core measures of stroke care quality to enable accurate international comparisons of care both in hospital and in the community (including structure, process, outcome measures and patient experience).

2. Assigning a named individual who is responsible for stroke quality improvement in each country or region.

3. Establishing national- and regional-level systems for assessing and accrediting stroke clinical services, providing peer support for quality improvement and making audit data routinely available to the general public.

4. Collecting patient-reported outcomes and longer term outcomes (e.g. six months and one year), covering both hospital and community care.

\section{Life after stroke}

\section{State of the art}

Life after stroke was not addressed specifically in the 2006 Helsingborg Declaration, ${ }^{3}$ because historically it has been largely considered as an aspect of rehabilitation: however, it has become increasingly apparent that this area merits recognition in its own right. Life after stroke is a large and overarching domain, and includes those who had a stroke in childhood (for whom it represents an entire lifespan) as well as those who had their stroke later in life. The domain includes consideration of family, friends and others who provide care and support for the stroke survivor, and whose own quality of life is also potentially affected; it covers stroke survivors living at home and in institutional homes.

Life after stroke has only been regarded as a separate entity in recent years. As a result, there are relatively few research studies covering the entire lifespan, and those that do tend to focus on reporting outcomes from rehabilitation interventions. Few of the current adult UK guidelines specifically address longer term stroke management, ${ }^{87}$ reflecting the paucity of evidence in this area. The World stroke Organization (WSO) has identified evaluating the best ways to address and improve life after stroke as a stated priority, although to date, this has not been adequately addressed. ${ }^{88}$ Similarly, the Global Stroke Bill of Rights ${ }^{89}$ also underlines the importance of providing longer term support. However, in reality, there is relatively little reference to life after stroke in national guidelines.

The few studies identifying long-term consequences after stroke report that around a third of stroke survivors are disabled, have poor post-stroke cognitive ability and poor mental health. From stroke survivors' own perspectives, surveys show that they also experience unmet needs in terms of communication, social relationships, loneliness, incontinence, fatigue and finances. ${ }^{90,91}$ It has been estimated that one in four strokes occur in people aged 65 and under, ${ }^{92}$ whose needs may also include support to return to work.

There is also parallel emerging evidence of the profound and long-term impact on carers; a 2013 report from the UK Stroke Association found that $80 \%$ of informal carers experienced anxiety, and that there were significant strains on relationships, with 1 in 10 people experiencing a breakdown of their relationship with their partner. ${ }^{93}$ There are also indications that there are growing numbers of young carers providing support for parents.

Qualitative research, and the Burden of Stroke in Europe Report, ${ }^{8}$ have documented the everyday plight of some survivors and their families. However, much of this information has not been collected systematically or rigorously, and there are countries for which there is no information available. Nevertheless, the illustrative texts paint a complex picture of life after stroke, and for many, it would seem that coping is tackled on an ongoing, daily basis with recognition that 'it's a new life; you have to adjust to what you can $d o$. ${ }^{8}$ There are also documented effects on family members ('...people are very lonely after they have lost these abilities [physical functions]. .... The families are very, very tired") and family members ("No one explained to my partner what it was going to be like moving forward, what the consequences might be, what they might not $\left.b e^{\prime}\right) .{ }^{8}$ Thus, although some survivors report positive experiences from changing their priorities after 
stroke, from focusing on relationships, and from deepened experiences, for many, there are significant day-to-day challenges. With an increasingly ageing population and more people surviving a stroke, it is therefore vital that stroke survivors and their families are empowered to optimise their quality of life and attain their maximum level of independence. This must be considered in relation to home, education and work, according to the stage of the person's life and their individual priorities. It is also important that this is considered in relation to other long-term conditions such as diabetes, hypertension, AF, depression, cognitive impairment, epilepsy, pain and spasticity. However, there is little robust evidence to support different approaches or models of care, and no agreement on what is meant by long-term survival: studies include people from six months to more than 20 years poststroke as 'long-term survivors'. Furthermore, no recognised scale exists to measure participation of stroke survivors in community activities.

The current literature lends itself to considering the issues surrounding life after stroke under four overlapping categories: health issues, activity issues, adjustment and wellbeing and information and support issues (Table 5). General principles surrounding these issues include: recognition by society of the worth and value of those with disabilities, including the importance of community integration and legislation; the need for a care plan after formal rehabilitation ends; the need for a seamless, structured and co-ordinated chain of support (still encompassing individual and tailored options) and the provision of services across the lifespan.

Life-long services required after stroke include access to ongoing diagnostics, therapy, medication, psychological support, rehabilitation and equipment as required, regular review and access to family support and self-management strategies. Figure 1 shows graphically how these might be integrated, as described in the 2007 UK National Stroke Strategy from the UK. ${ }^{94}$

\section{State of current services}

There are relatively few robust data available across Europe on longer term management and care after stroke. Where data exist, collection has been relatively unsystematic, and the service data are therefore both patchy and anecdotal; little attention has been paid to developing, let alone evaluating, longer term care. Stroke survivors seem ill prepared for the transition from rehabilitation, and many studies report survivors and their carers feeling 'abandoned'. ${ }^{95}$ There is evidence of six-month reviews being conducted in some European countries, but there is a conspicuous reduction in data from survivors or their carers beyond this
Table 5. Life after stroke issues. ${ }^{a}$

\section{Health issues}

- Specific post-stroke disabilities, e.g. arm function, vision, dysphagia, spasticity (need for 'top up' in rehabilitation; regular review and equipment)

- Hidden post-stroke deficits, e.g. psychological, cognitive, depression, anxiety, communication, fatigue and incontinence

- Co-existing conditions including frailty and dementia (medication implications) and associated with old age, e.g. hearing

- Need for links to secondary prevention in primary care, e.g. medication, diet and exercise

- Emergence of sequelae over time, e.g. epilepsy and depression

- End of life care

Activity issues

- Meaningful activities including leisure, holidays and play

- Vocational support - getting back to work or education

- Mobility including driving, transportation and access

- Vocational support - getting back to work/education

- Role in family and society (issues regarding relationships and divorce)

- Friendships - making and keeping friends

- Key life transitions, e.g. entering school, discharge from rehabilitation

- Communication

Adjustment and wellbeing issues

- Coming to terms with new life

- Specific individual issues, such as sex, sleep, fatigue and confidence

- Happiness/life satisfaction/loneliness

- Grief and adjustment of parents/carers for changed future prospects

- Emotional, behavioural and psychosocial domains of wellbeing

- Environmental including nursing home/residential care Information and support issues for individual and carers/parents

- Self-management (includes parent education to support their child)

- Advocacy psychological and emotional support

- Communication-including aphasia friendly and culturally sensitive literature

- Cognitive support, e.g. memory and concentration

- Financial support including benefits. Issues re-additional costs of stroke

- Long-term support groups, peer support and volunteering

- Community integration - loneliness/isolation

- Practical help with specific tasks, e.g. housework and shopping

- Carer support (including children-parents and siblings; parents-siblings, relationship support for partners) and respite care

- Specific support, e.g. around return to work.

- IT access support; web-based interventions, tele- rehabilitation, podcasts on life after stroke/audiobooks, virtual reality-based support

- Sharing of relevant information across health, education, work and social care with the appropriate consents of the patient and carers

- Proactive review

${ }^{\text {aT }}$ This is not an exhaustive list, but merely an indication of the breadth and depth of issues faced across the lifespan after stroke: many issues will overlap between categories. 


\section{The range ot support someone may need after a stroke}

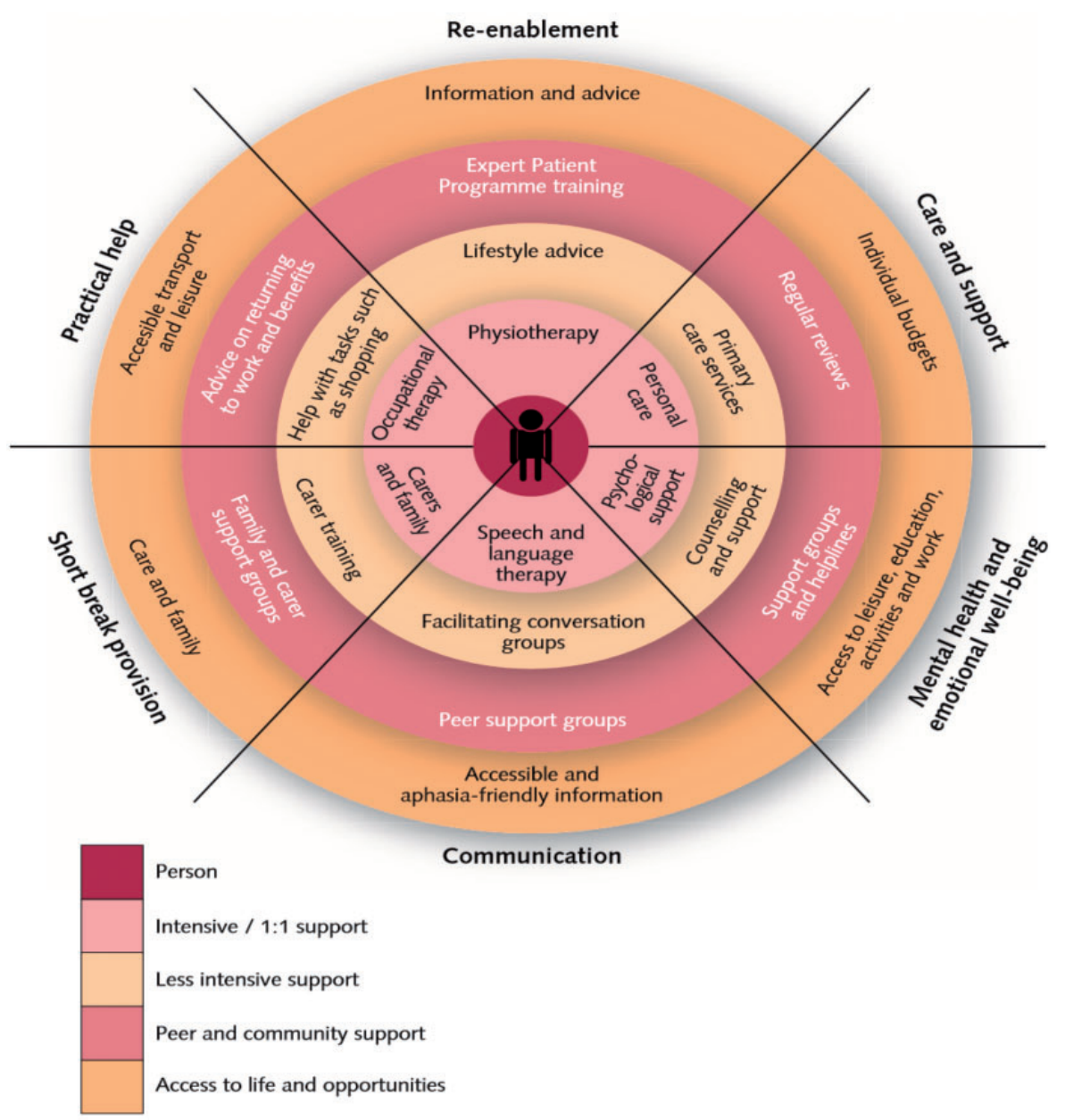

Figure I. Range of support needed after stroke. ${ }^{94 *}$ *This model was worded specifically for adults.

time. Similarly, there is no Europe-wide agreed model for the long-term support for children or young people who have had a stroke. Their needs, and those of their families, are typically provided by community services that usually also cover cerebral palsy, acquired brain injury and allied neurological conditions. However, it is rare for a child to receive intensive residential rehabilitation services after discharge from hospital. Families may have access to specialist tertiary level support around the time of diagnosis, but continuing access to this support varies and typically tapers off over time. Overall, there appears to be little evidence of holistic and coordinated support. There is no pathway for survivors, who often find themselves on parallel paths for other conditions (e.g. depression and diabetes); longer term review may enable stroke survivors and their carers to better follow their individual disease management plan. Most importantly, there is no model of what best care looks like following discharge from specialist services. The large and growing number of stroke survivors with long-term needs must be addressed; we need to develop minimum standards of care and measure these.

There is a growing interest in collecting patientreported outcome measures (PROMs) data that would best reflect patients' views about their care and provide additional information beyond the traditional clinician-reported measures. Patient representatives have played an important role in the development of these measures. The American Heart Association has advocated broader use of PROMs in both clinical practice and research. ${ }^{96}$ Similarly, the Swedish Stroke Register ${ }^{97}$ has gradually broadened to more fully cover the quality of services and late outcomes after stroke, through follow-up at three months and at one year after the event. ${ }^{98}$ There is an emphasis on PROMs 
relating to ADL, self-perceived health, health-related quality of life, mood, pain, satisfaction with stroke services, rehabilitation and community support; all of these are important for stroke survivors. Questions about the need for support for the carer may also be included.

\section{Research and development priorities}

- What are the experiences and needs of stroke survivors at different times during their lifespan?

- What are the needs of different cohorts of stroke survivors? This needs to include patient- and carerreported outcomes, and to address the challenges of stroke survivors with multiple morbidities, and their carers, in order to enable the design of optimal care pathways.

- What would a model of best care and long-term support look like? This should include the provision of three to six-month and annual reviews and specific roles to provide holistic coordinated support.

- What products and services (digital and physical) would support self-management, community integration, education and healthcare?

- How can high-quality information and training to help non-specialist staff, especially social care staff, be targeted? It is envisaged that this will involve research around staffing levels, core competencies and the involvement of non-governmental and non-profit-making bodies such as charities and voluntary groups.

\section{Targets for 2030}

1. Appointing government-level individuals or teams responsible for championing life after stroke and ensuring that national stroke plans address survivors' and their families' long-term unmet needs. Minimum standards set for what every stroke survivor should receive regardless of where they live.

2. Formalising the involvement of stroke survivors and carers, and their associations, in identifying issues and solutions to enable the development of best patient and support practices.

3. Establishing, through national stroke care plans, the support that will be provided to stroke survivors, regardless of their place of residence and socioeconomic status.

4. Supporting self-management and peer support for stroke survivors and their families, by backing stroke support organisations

5. Supporting the implementation of digitally-based stroke self-help information and assistance systems.

\section{Prioritised research areas for translational stroke research}

\section{State of the art}

Clinical discoveries have resulted in huge improvements in the management and treatment of acute ischaemic stroke patients. There have been advances in understanding the pathophysiology of stroke and chronic cerebrovascular diseases, ${ }^{99}$ but the goal of translating this knowledge into successful treatments has not been achieved. The efficacy of mechanical thrombectomy increases the potential for successful translation of neuroprotective agents. Technological developments offer new opportunities for deciphering pathophysiological processes underlying cerebrovascular diseases, identifying novel targets for drug therapies and drug repurposing. There is also increasing interest in personalised medicine approaches utilising genomics, imaging and other biomarkers for stratification of stroke patients. However, novel opportunities run the risk failing in translation, unless accompanied by conceptual changes in the way research is performed.

Bridging the 'translational gap' between basic and clinical stroke research is critical for the development of effective treatments. Key requirements here include improved networking between laboratory scientists and clinicians, better experimental designs and the development of more relevant experimental models that mirror the complexity of human diseases. ${ }^{100}$ The disappointing outcome of translation in the last decades demands novel methodological strategies. ${ }^{101,102}$

The following sections highlight major research topics where recent advances offer new opportunities for translational studies, where there is urgent need for therapeutic strategies, or both. Next, we analyse critical problems in the current pipeline of translational research and suggest action points aimed at changing conventional practice by designing novel strategies to tackle translational bench-to-beside research.

\section{Key translational research topics}

Reperfusion. Current optimal treatment for acute ischaemic stroke is recanalisation with intravenous tPA, endovascular treatment or both. However, microvascular reperfusion is not always achieved, not all patients benefit from endovascular treatment, and many patients are not eligible for reperfusion therapies.

Improving tissue reperfusion: Strategies designed to dissolve thrombi or prevent thrombus formation locally in the capillary bed should be investigated, either alone or in combination with mechanical recanalisation. Recent research has identified cellular and 
molecular targets that may help achieve optimal reperfusion according to clot composition and location.

Reducing reperfusion injury: Secondary complications of reperfusion therapies, such as haemorrhagic transformation, should be minimised. Complications due to previous treatments, such as antiplatelet drugs and anticoagulants, and the effective use of antidotes, require further investigation. Current experimental evidence suggests that oxidative or nitrosative stress and thrombo-inflammation ${ }^{103}$ impair the benefits of reperfusion, but this concept awaits translation to the clinic.

\section{Protective drugs for treatment and prevention.} Neuroprotection: Translation of drug treatments to the new clinical recanalisation scenario offers opportunities for novel and old drug candidates that have failed in the past when administered too late or in the absence of reperfusion. ${ }^{104}$ The concept of neuroprotection has evolved because protection is needed for functional units composed of neurons, glial cells, pericytes, macrophages and the vasculature. Approaches to protecting the white matter, which is under-represented in most animal models of stroke, need further development.

Vascular protection: Protecting the vasculature is critical in ischaemia and reperfusion since the vasculature plays a major role in propagating injury through both acute and chronic vascular dysfunction. A good collateral circulation can reduce infarct size and secondary growth by supplying blood to the penumbra, but we still do not know how to effectively modulate collateralisation for preventive or acute therapy. Detailed investigation, using advanced imaging techniques and vascular biology research, is required to obtain data on vascular integrity, haemodynamic responses and blood-brain barrier (BBB) function.

Inflammatory and immune responses. Promising cellular and molecular inflammatory mechanisms, with potential impacts on acute stroke outcomes and post-stroke recovery, have been identified. ${ }^{105}$ However, substantial basic research is required for a more comprehensive understanding of the complex neuro-immune interactions before translational strategies are attempted. Chronic inflammation has emerged as an important pathogenetic mechanism in neurodegenerative and chronic cerebrovascular diseases, and may be relevant for cognitive decline and dementia. ${ }^{106}$ Stroke triggers a multiphasic systemic inflammatory response that involves neural signals and soluble mediators, including cytokines, vasoactive substances, alarmins and acute phase proteins, followed by an inhibition of immune responses that increases the risk of poststroke infection. Imbalance of peripheral immune homeostasis after stroke represents a promising therapeutic target that requires investigation. Repurposing of available drugs in clinical use for other inflammatory conditions could improve the translational efficacy in testing anti-inflammatory therapies for stroke. ${ }^{107}$

Intracerebral haemorrhage and SAH. New cellular and molecular processes, including inflammation, are recognised as critical players for brain damage after ICH. ${ }^{108} \mathrm{~A}$ genetic contribution to these processes has been identified and requires exploration in experimental models, as well as translation to pharmaceutical targets. Research in $\mathrm{ICH}$ is particularly needed given the devastating clinical consequences of $\mathrm{ICH}$ and the current lack of treatment options for this condition. Appropriate experimental models of $\mathrm{ICH}$ and $\mathrm{SAH}$, and models mimicking aneurysm rupture, are required. Mechanisms of early ischaemic damage preceding vasospasm after $\mathrm{SAH}$ should be investigated.

SVD. Specific treatments for established SVD are lacking, despite its enormous health importance as the cause of $20 \%$ of ischaemic strokes, and a major cause of intracerebral haemorrhage, vascular cognitive impairment or dementia. Incomplete understanding of SVD pathogenesis and a lack of appropriate animal models are obstacles to progress. Large-scale studies of systems biology can provide insights into the underlying mechanisms of complex diseases and responses to treatment. ${ }^{109}$ Key priorities in this area include: better understanding of the pathogenic contribution of endothelial dysfunction or BBB disruption, inflammation and hemodynamic factors; understanding the role of proteins and pathways involved in monogenic forms of SVD and (multifactorial) sporadic SVD $;{ }^{110}$ understanding the mechanisms leading to cognitive impairment and deciphering the intricate relationship with Alzheimer's disease; designing protective approaches to reduce progression of white matter damage; expanding the identification of genetic and other molecular biomarkers and translating the new putative targets discovered through genetic, molecular and cellular biology data into diseasemodifying therapies.

Functional recovery and rehabilitation. A key objective is to expand our knowledge on functional recovery mechanisms and potential therapeutic targets to enhance the effects of physical rehabilitation in the chronic phase after stroke. Research is needed on neuronal plasticity and network recovery, and their interaction with delayed pathophysiological mechanisms such as neuroinflammation, apoptosis, neurogenesis and angiogenesis. This will require the adoption of methodology from stem cell research, gene therapy, optogenetics, 
non-invasive brain stimulation and other fields. The methodology for evaluating functional recovery in experimental stroke models should be improved.

\section{Key research pipeline priorities}

A critical question is how translational research should be performed if it is to be successful. Despite promising novel advances, the field risks propagating the same methodological errors that have blocked translation to the present day. A number of crucial strategic steps are proposed to overcome the block in translation, and action points relating to these are summarised in Table 6.

Exploratory versus confirmatory studies. Preclinical research has traditionally lacked genuine confirmatory studies for testing treatment efficacy. Instead, exploratory studies identifying and investigating novel molecular or cellular pathways and mechanisms were frequently combined with therapeutic experiments. However, these are often hampered by insufficient statistical power and poor designs, resulting in low reproducibility.

Preclinical, confirmatory studies as an intermediate translational step. Clinical trials based on preclinical target identification and drug development have typically relied on a few small-scale, single-centred and underpowered studies. As a result, current translational efforts represent an enormous leap from small exploratory studies to large, confirmatory trials in a highly variable human disease. This huge gap requires an intermediate step to improve the translational reliability and solve the problem of lack of replication.

Improve experimental modelling. An important reason why translation has failed so far is that current experimental modelling lacks internal, external and construct validity. Experiments are mostly performed in young, male, genetically identical rodents, housed in artificial pathogen-free conditions, under anaesthesia. As a result, they may not accurately reflect the diverse conditions encountered in clinical medicine.

Adopt a 'Team Science' approach. Large-scale collaborations with a 'Team Science' approach are needed to guide further development in translational research. Clinicians should be partnering with basic researchers by specifying research needs and contributing to preclinical study design from a clinical perspective. Initiatives for large-scale preclinical multicentric trials using, as much as possible, protocols commonly accepted for clinical trials, have started, but the utility of this tool remains to be shown.

Improve efficacy of early stage clinical trials. Early-stage clinical trials represent an intermediate step between preclinical drug development and large-scale clinical trials. As such, the designs should keep up with the pace of preclinical target identification, be sufficiently sensitive to test novel approaches in a stratified, optimal target population and when possible seek a genetic rationale for drug effects.

Table 6. Proposed strategic steps for translational research in stroke, with related action points.

\begin{tabular}{ll}
\hline Strategic step & Action points \\
\hline Exploratory versus confirmatory studies & - High-quality basic research with: \\
& - state-of-the art, rigorous, methodology \\
& - transparency \\
& - data availability (e.g. deposition of protocols/data in public repositories) \\
& - avoidance of publication bias \\
Preclinical confirmatory studies as an & - Separation of discovery and confirmation \\
intermediate translational step & - Confirmation before undertaking clinical studies \\
& - Preregistration \\
& - Pre-planned study designs and analyses \\
Improve experimental modelling & - Publication of all results (including negative results) \\
& - Reduce bias \\
& - Increase power \\
& - Include comorbidities \\
Change to a larger 'team' concept & - Less standardisation, more variability (e.g. genetics, \\
Improve efficacy of early stage clinical trials & - - Establishing 'Team Science' in stroke research \\
& - Regulations should be more proportionate to the risks of the trial \\
& - Carefully stratify patients for clinical trial inclusion, with the perspective \\
& of developing personalised treatment approaches
\end{tabular}




\section{Involvement of various parties}

Commitment is needed from EU and national funding agencies to invest in stroke research in a proportionate manner to the magnitude and prevalence of the health problem. Strategies designed to validate findings from exploratory research require a joint effort that exceeds the capacity of individual projects or small sporadic collaborative endeavours. Strong independent institutional support is required to make the transition from classical designs to a novel concept of organised research structures and data validation that aims to facilitate reliable translation of preclinical findings to clinical practice. The pharmaceutical industry should also be engaged in this process: involvement of pharmaceutical companies could be increased by facilitating exchanges between academic and pharmaceutical research at the point of transition from exploratory to confirmatory preclinical studies. Finally, researchers must disseminate their findings to bring stroke research closer to patient associations and the general population.

\section{Targets for 2030}

1. Achieving an organisational framework by implementing confirmatory preclinical research through 'Team Science' and by providing novel instruments for advanced experimental designs to increase validity.

2. Establishing and implementing guidelines for preclinical trials of new treatments to maximise the success of clinical translation.

3. Focusing experimental stroke research on identification of new treatable targets with high translational potential that lead to successful clinical trials by 2030 .

\section{Conclusions}

ESAP provides a comprehensive overview of the current state of stroke management, as well as specific targets and research and development priorities for stakeholders. The ESAP Implementation Plan will be drafted after having assessed an updated epidemiological report on stroke incidence, prevalence and mortality and receiving detailed and reliable data from quality registries from national stroke and patient societies. It is anticipated that the progress towards the targets and research and development priorities laid out in this paper will be reviewed in 2021 and 2024, with a midterm review scheduled for 2024. The extent to which the targets have been achieved will be reviewed in 2030 .

\section{Acknowledgements}

We are grateful to the ESO Head Office, Basel, Switzerland, and especially we want to thank Luzia Balmer for excellent secretarial assistance. We are grateful to the Carl von Siemens Stiftung for providing the venue for the Munich workshop. Editorial support in the preparation of this paper, funded by the ESO, was provided by Michael Shaw PhD (MScript Ltd, Hove, UK).

\section{Declaration of Conflicting Interests}

The author(s) declared the following potential conflicts of interest with respect to the research, authorship, and/or publication of this article: Bo Norrving has received honoraria for DSMB work for Astra Zeneca and Bayer; Valeria Caso has received honoraria for participation in advisory boards, consulting fees, or as a symposium speaker for DaiichiSankyo, Bayer, BMS, Pfizer, Amgen and BoehringerIngelheim; Charlotte Cordonnier was investigator in clinical trials for Pfizer, Astra-Zeneca, Boehringer-Ingelheim, Daichii-Sankyo, Servier, participated to advisory boards for Bayer, Medtronics, Daichii-Sankyo, BMS-Pfizer (fees were paid to research account from Adrinord or the Lille University Hospital. No personal funding); Alla Guekht has received grants/research support from EVER NeuroPharma and Takeda; Jon Barrick reported that SAFE receives educational grants and project sponsorship from Commercial organisations; Richard Hobbs has received consultancy fees from Boehringer-Ingelheim, Bayer and Pfizer/BMS; Patrik Michel reports research grants from Boehringer-Ingelheimand BMS; speaker fees from Boehringer-Ingelheim, Bayer, Daiichi-Sankyo, Medtronic and Amgen; consulting fees from Medtronic, Astra-Zeneca and Amgen, and honoraria from scientific advisory boards from Boehringer-Ingelheim, Pfizer and BMS (all fees paid goes to CHUV, no personal fees); Peter Rothwell has received honoraria from Bayer for Executive Committee of the ARRIVE Trial; Christian Gerloff has received fees from Abbott, Acticor Biotech, Amgen, Bayer Vital, Boehringer Ingelheim, Prediction Biosciences, Sanofi Aventis; Aleksandra Pavlovic has received travel grants by Richter Gedeon and PharmaSwiss; Heinrich Audebert has received speaker honoraria and consultancy fees by Boehringer Ingelheim, Pfizer, BMS, Bayer, Lundbeck, Roche, Sanofi, Daiichi Sankyo, Ever Pharma and Novo Nordisk; Urs Fischer has received consultancy fees from Medtronic and Stryker; Kennedy R Lees has received fees from Applied Clinical Intelligence, Boehringer Ingelheim, EVER NeuroPharma, Hilicon, Nestlé, Parexel; Mikael Mazighi has received fees from BMS-Pfizer, Boehringer, Servier, Astra Zeneca, Medtronic, Acticor, Amgen; Bart van der Worp has received fees from Boehringer Ingelheim and Bayer; Philip Bath has received fees from Diamedica, Nestle, Phagenesis, ReNeuron and report share ownership in Diamedica, Platelet Solutions; Hans-Christoph Diener has received honoraria for participation in clinical trials, 
contribution to advisory boards or oral presentations from Abbott, Achelios, Allergan, AstraZeneca, Bayer Vital, BMS, Boehringer Ingelheim, CoAxia, Corimmun, Covidien, Daiichi-Sankyo, D-Pharm, Fresenius, GlaxoSmithKline, Janssen-Cilag, Johnson \& Johnson, Knoll, Lilly, MSD, Medtronic, MindFrame, Neurobiological Technologies, Novartis, Novo-Nordisk, Paion, Parke-Davis, Pfizer, Portola, Sanofi-Aventis, Schering-Plough, Servier, Solvay, St. Jude, Syngis, Talecris, Thrombogenics, WebMD Global, Wyeth and Yamanouchi and financial support for research projects from AstraZeneca, GSK, Boehringer Ingelheim, Lundbeck, Novartis, Janssen-Cilag, Sanofi-Aventis, Syngis and Talecris; Jean-Louis Mas has received fees from Bayer, Boerhinger-Ingelheim, Bristol Myers Squibb and DaiichiSankyo; Maurizio Paciaroni has received fees from Aspen, Sanofi-Aventis, Boehringer Ingelheim, Bayer, Bristol Meyer Squibb, Daiiki Sankyo and Pfizer; Katharina S Sunnerhagenhas received fees from Allergan; Jesse Dawson has received fees from Bayer, Bristol Myers Squibb and Boeringher Ingelheim; Didier Leys was investigator in clinical trials for Pfizer, Astra-Zeneca, Sanofi Aventis, BoehringerIngelheim, Daichii-Sankyo, Servier, participated to advisory boards for Bayer, BMS-Pfizer, Astrazeneca, BoeringherIngelheim, (fees were paid to research account from Adrinord or the Lille University Hospital. No personal funding); Andreas Luft has received fees from Hocoma AG; Yannick Béjot has received fees for participation in advisory boards, consulting fees, or as a symposium speaker for AstraZeneca, Daiichi-Sankyo, MSD, Bayer, Medtronic, BMS, Pfizer, Amgen and Boehringer-Ingelheim; Hugh Markus has received fees from Astra Zeneca for preparation of educational material. The other authors report no conflicts.

\section{Funding}

The author(s) disclosed receipt of the following financial support for the research, authorship, and/or publication of this article: The costs for the preparation of the ESAP were borne by the ESO. There was no private sector involvement in the preparation and development of the ESAP.

\section{Ethical approval}

Ethical approval was not necessary for the work described in this paper.

\section{Informed consent}

Not applicable.

\section{Guarantor}

A special guarantor does not exist. The working group has jointly developed the manuscript.

\section{Contributorship}

All listed authors have contributed to the preparation and writing of the manuscript. All authors reviewed and approved the final version of the manuscript.

\section{References}

1. United Nations, Department of Economic and Social Affairs, Population Division (2017). World Population Ageing 2017 - Highlights (ST/ESA/SER.A/397) (accessed 11 October 2018).

2. Aboderin I, Venables G and Asplund K. Stroke management in Europe. J Intern Med 1996; 240: 173-180.

3. Kjellström T, Norrving $B$ and Shatchkute A. Helsingborg Declaration 2006 on European Stroke Strategies. Cerebrovasc Dis 2007; 23: 229-241.

4. O’Donnell MJ, Chin SL, Rangarajan S, et al. Global and regional effects of potentially modifiable risk factors associated with acute stroke in 32 countries (INTERSTROKE): a case-control study. Lancet 2016; 388: 761-775.

5. Piepoli MF, Hoes AW, Agewall S, et al. 2016 European Guidelines on cardiovascular disease prevention in clinical practice: the Sixth Joint Task Force of the European Society of Cardiology and Other Societies on Cardiovascular Disease Prevention in Clinical Practice (constituted by representatives of 10 societies and by invited experts) developed with the special contribution of the European Association for Cardiovascular Prevention \&amp; Rehabilitation (EACPR). Eur Heart $J$ 2016; 37: 2315-2381.

6. Goff DC Jr, Lloyd-Jones DM, Bennett G, et al. 2013 ACC/AHA guideline on the assessment of cardiovascular risk: a report of the American College of Cardiology/ American Heart Association Task Force on Practice Guidelines. Circulation 2014; 129: S49-S73.

7. GBD 2016 Risk Factors Collaborators. Global, regional, and national comparative risk assessment of 84 behavioural, environmental and occupational, and metabolic risks or clusters of risks, 1990-2016: a systematic analysis for the Global Burden of Disease Study 2016. Lancet 2017; 390: 1345-1422.

8. Stevens E, McKevitt C, Emmett E, et al. The burden of stroke in Europe. London: Stroke Alliance for Europe (SAFE), www.strokeeurope.eu/downloads/ TheBurdenOfStrokeInEuropeReport.pdf (2017, accessed 19 April 2018).

9. European Guidelines on CVD Prevention in Clinical Practice (2012). European Heart Journal 2012; 33: 1635-1701.

10. Beishuizen CR, Stephan BC, van Gool WA, et al. Webbased interventions targeting cardiovascular risk factors in middle-aged and older people: a systematic review and meta-analysis. J Med Internet Res 2016; 18: e55.

11. Rose G. Strategy of prevention: lessons from cardiovascular disease. Br Med J (Clin Res Ed) 1981; 282: 1847-1851.

12. World Health Organization. Global action plan for the prevention and control of non-communicable diseases 2013-2020, http://africahealthforum.afro.who.int/ 
IMG/pdf/global_action_plan_for_the_prevention_and_ control_of_ncds_2013-2020.pdf (accessed 4 May 2018).

13. Kotseva $\mathrm{K}$, Wood D, De Backer G, et al. EUROASPIRE III. Management of cardiovascular risk factors in asymptomatic high-risk patients in general practice: cross-sectional survey in 12 European countries. Eur J Cardiovasc Prev Rehabil 2010; 17: 530-540.

14. World Health Organization. WHO global report on trends in prevalence of tobacco smoking 2015. http:// apps.who.int/iris/bitstream/10665/156262/1/978924156 4922_eng.pdf?ua.1 (accessed 11 October 2018).

15. https://ec.europa.eu/eurostat/statistics-explained/index. php/Tobacco_consumption_statistics (accessed 11 October 2018).

16. Lewey J, Shrank WH, Bowry AD, et al. Gender and racial disparities in adherence to statin therapy: a meta-analysis. Am Heart J 2013; 165: 665-678.

17. Freedman B, Camm J, Calkins H, et al. Screening for atrial fibrillation: a report of the AF-SCREEN international collaboration. Circulation 2017; 135: 1851-1867.

18. Waje-Andreassen U, Nabavi DG, Engelter ST, et al. European Stroke Organisation certification of stroke units and stroke centres. Eur Stroke J 2018; 3: 220-226.

19. Hillmann S, Wiedmann S, Fraser A, et al. Temporal changes in the quality of acute stroke care in five national audits across Europe. Biomed Res Int 2015; 2015: 432497.

20. Mellon L, Hickey A, Doyle F, et al. Can a media campaign change health service use in a population with stroke symptoms? Examination of the first Irish stroke awareness campaign. Emerg Med J 2014; 31: 536-540.

21. Cadilhac DA, Kim J, Lannin NA, et al. National stroke registries for monitoring and improving the quality of hospital care: a systematic review. Int J Stroke 2016; 11: $28-40$.

22. Wellwood I, Wu O, Langhorne P, et al. Developing a tool to assess quality of stroke care across European populations: the EROS quality assessment tool. Stroke 2011; 42: 1207-1211.

23. Rudd M, Buck D, Ford GA, et al. A systematic review of stroke recognition instruments in hospital and prehospital settings. Emerg Med $J$ 2016; 33: 818-822.

24. Watkins CL, Leathley MJ, Jones SP, et al. Training emergency services' dispatchers to recognise stroke: an interrupted time-series analysis. BMC Health Serv Res 2013; 13: 318.

25. Fothergill RT, Williams J, Edwards MJ, et al. Does use of the recognition of stroke in the emergency room stroke assessment tool enhance stroke recognition by ambulance clinicians? Stroke 2013; 44: 3007-3012.

26. Eriksson M, Glader EL, Norrving B, et al. Acute stroke alert activation, emergency service use, and reperfusion therapy in Sweden. Brain Behav 2017; 7: e00654.

27. Faiz KW, Sundseth A, Thommessen B, et al. Prehospital delay in acute stroke and TIA. Emerg Med $J$ 2013; 30: 669-674.

28. Ebinger M, Kunz A, Wendt M, et al. Effects of golden hour thrombolysis: a Prehospital Acute Neurological
Treatment and Optimization of Medical Care in Stroke (PHANTOM-S) substudy. JAMA Neurol 2015; 72: 25-30.

29. Reiner-Deitemyer V, Teuschl Y, Matz K, et al. Helicopter transport of stroke patients and its influence on thrombolysis rates: data from the Austrian Stroke Unit Registry. Stroke 2011; 42: 1295-1300.

30. Lahr MM, Luijckx GJ, Vroomen PC, et al. Proportion of patients treated with thrombolysis in a centralized versus a decentralized acute stroke care setting. Stroke 2012; 43: 1336-1340.

31. Bray BD, Ayis S, Campbell J, et al. Associations between the organisation of stroke services, process of care, and mortality in England: prospective cohort study. BMJ 2013; 346: f2827.

32. Stroke Unit Trialists' Collaboration. Organised inpatient (stroke unit) care for stroke. Cochrane Database Syst Rev 2013; 9: CD000197.

33. Wardlaw JM, Seymour J, Cairns J, et al. Immediate CT scanning of acute stroke is cost effective and improves quality of life. Stroke 2004; 35: 2477-2483.

34. Fearon P and Langhorne P. Early Supported Discharge Trialists. Services for reducing duration of hospital care for acute stroke patients. Cochrane Database Syst Rev 2012; 9: CD000443.

35. Langhorne $\mathrm{P}$ and Baylan S. Early Supported Discharge Trialists. Early supported discharge services for people with acute stroke. Cochrane Database Syst Rev 2017; 7: CD000443.

36. Audebert HJ, Schultes K, Tietz V, et al. Long-term effects of specialized stroke care with telemedicine support in community hospitals on behalf of the Telemedical Project for Integrative Stroke Care (TEMPiS). Stroke 2009; 40: 902-908.

37. Urimubenshi G, Langhorne P, Cadilhac DA, et al. Association between patient outcomes and key performance indicators of stroke care quality: a systematic review and meta-analysis. Eur Stroke J 2017; 2: 287-307.

38. Bray BD, Cloud GC, James MA, et al. Weekly variation in health-care quality by day and time of admission: a nationwide, registry-based, prospective cohort study of acute stroke care. Lancet 2016; 388: 170-177.

39. Bray BD, Campbell J, Cloud GC, et al. Bigger, faster? Associations between hospital thrombolysis volume and speed of thrombolysis administration in acute ischemic. Stroke 2013; 44: 3129-3135.

40. Aguiar de Sousa D, von Martial R, Abilleira S. Access to and delivery of acute ischaemic stroke treatments: a survey of national scientific societies and stroke experts in 44 European countries. Eur Stroke J 2018; epub: DOI: $10.1177 / 2396987318786023$.

41. Lees KR, Bluhmki E, von Kummer R, et al. Time to treatment with intravenous alteplase and outcome in stroke: an updated pooled analysis of ECASS, ATLANTIS, NINDS, and EPITHET trials. Lancet 2010; 375: 1695-1703.

42. Emberson J, Lees KR, Lyden P, et al. Effect of treatment delay, age, and stroke severity on the effects of intravenous thrombolysis with alteplase for acute ischaemic 
stroke: a meta-analysis of individual patient data from randomised trials. Lancet 2014; 384: 1929-1935.

43. Saver JL, Goyal M, van der Lugt A, et al. Time to treatment with endovascular thrombectomy and outcomes from ischemic stroke: a meta-analysis. JAMA 2016; 316: 1279-1288.

44. Reinink H, de Jonge JC, Bath PM, et al. PRECIOUS: PREvention of Complications to Improve OUtcome in elderly patients with acute Stroke. Rationale and design of a randomised, open, phase III, clinical trial with blinded outcome assessment. Eur Stroke J 2018; 3; 291-298.

45. Johnston SC, Easton JD, Farrant M, et al. Clopidogrel and aspirin in acute ischemic stroke and high-risk TIA. $N$ Engl J Med 2018 379: 215-225.

46. Berkhemer OA, Fransen PS, Beumer D, et al. A randomized trial of intraarterial treatment for acute ischemic stroke. $N$ Engl J Med 2015; 372: 11-20.

47. Goyal M, Demchuk AM, Menon BK, et al. Randomized assessment of rapid endovascular treatment of ischemic stroke. $N$ Engl J Med 2015; 372: 1019-1030.

48. Saver JL, Goyal M, Bonafe A, et al. Stent-retriever thrombectomy after intravenous t-PA vs. t-PA alone in stroke. $N$ Engl J Med 2015; 372: 2285-2295.

49. Campbell BC, Mitchell PJ, Kleinig TJ, et al. Endovascular therapy for ischemic stroke with perfusion-imaging selection. $N$ Engl J Med 2015; 372: 1009-1018.

50. Jovin TG, Chamorro A, Cobo E, et al. Thrombectomy within 8 hours after symptom onset in ischemic stroke. N Engl J Med 2015; 372: 2296-2306.

51. Thomalla G, Simonsen CZ, Boutitie F, et al. MRIguided thrombolysis for stroke with unknown time of onset. $N$ Engl J Med 2018; 379: 611-622.

52. Hofmeijer J, Kappelle LJ, Algra A, et al. Surgical decompression for space-occupying cerebral infarction (the Hemicraniectomy After Middle Cerebral Artery infarction with Life-threatening Edema Trial [HAMLET]): a multicentre, open, randomised trial. Lancet Neurol 2009; 8: 326-333.

53. Juttler E, Unterberg A, Woitzik J, et al. Hemicraniectomy in older patients with extensive middle-cerebral-artery stroke. $N$ Engl J Med 2014; 370: 1091-1100.

54. Anderson CS, Chalmers J and Stapf C. Blood-pressure lowering in acute intracerebral hemorrhage. $N$ Engl $J$ Med 2013; 369: 1274-1275.

55. Steiner T, Al-Shahi Salman R, Beer R, et al. European Stroke Organisation (ESO) guidelines for the management of spontaneous intracerebral hemorrhage. Int $J$ Stroke 2014; 9: 840-855.

56. Hemphill JC 3rd, Greenberg SM, Anderson CS, et al. Guidelines for the management of spontaneous intracerebral hemorrhage: a guideline for healthcare professionals from the American Heart Association/American Stroke Association. Stroke 2015; 46: 2032-2060.

57. Steiner T, Juvela S, Unterberg A, et al. European Stroke Organization guidelines for the management of intracranial aneurysms and subarachnoid haemorrhage. Cerebrovasc Dis 2013; 35: 93-112.

58. Connolly ES Jr, Rabinstein AA, Carhuapoma JR, et al. Guidelines for the management of aneurysmal subarachnoid hemorrhage: a guideline for healthcare professionals from the American Heart Association/American Stroke Association. Stroke 2012; 43: 1711-1737.

59. Geurts M, Macleod MR, van Thiel GJ, et al. End-of-life decisions in patients with severe acute brain injury. Lancet Neurol 2014; 13: 515-524.

60. Lawrence M, Pringle J, Kerr S, et al. Multimodal secondary prevention behavioral interventions for TIA and stroke: a systematic review and meta-analysis. PLoS One 2015; 10: e0120902.

61. Deijle IA, Van Schaik SM, Van Wegen EE, et al. Lifestyle interventions to prevent cardiovascular events after stroke and transient ischemic attack: systematic review and meta-analysis. Stroke 2017; 48: 174-179.

62. D'Isabella NT, Shkredova DA, Richardson JA, et al. Effects of exercise on cardiovascular risk factors following stroke or transient ischemic attack: a systematic review and meta-analysis. Clin Rehabil 2017; 31: 1561-1572.

63. Katsanos AH, Filippatou A, Manios E, et al. Blood pressure reduction and secondary stroke prevention: a systematic review and metaregression analysis of randomized clinical trials. Hypertension 2017; 69: 171-179.

64. Niu PP, Guo ZN, Jin H, et al. Antiplatelet regimens in the long-term secondary prevention of transient ischaemic attack and ischaemic stroke: an updated network meta-analysis. BMJ Open 2016; 6: e009013.

65. Koskinas KC, Siontis GCM, Piccolo R, et al. Effect of statins and non-statin LDL-lowering medications on cardiovascular outcomes in secondary prevention: a meta-analysis of randomized trials. Eur Heart J 2018; 39: 1172-1180.

66. Lokuge K, de Waard DD, Halliday A, et al. Meta-analysis of the procedural risks of carotid endarterectomy and carotid artery stenting over time. Br J Surg 2018; 105: 26-36.

67. López-López JA, Sterne JAC, Thom HHZ, et al. Oral anticoagulants for prevention of stroke in atrial fibrillation: systematic review, network meta-analysis, and cost effectiveness analysis. BMJ 2017; 359: j5058.

68. Niu X, Ou-Yang G, Yan PF, et al. Closure of patent foramen ovale for cryptogenic stroke patients: an updated systematic review and meta-analysis of randomized trials. J Neurol 2018 265: 1259-1268.

69. World Health Organization. World Report on Disability 2011. Geneva: World Health Organization, 2011. Rehabilitation. www.ncbi.nlm.nih.gov/books/NBK30 4081/ (accessed 19 April 2018).

70. United Nations Division for Social Policy and Development. Convention on the Rights of Persons with Disabilities (CRPD), www.un.org/development/ desa/disabilities/convention-on-the-rights-of-personswith-disabilities.html (accessed 19 April 2018).

71. World Health Organization. Rehabilitation 2030: a call for action, www.who.int/disabilities/care/rehab-2030/ en/ (2017, accessed 24 April 2018). 
72. Forster A, Brown L, Smith J, et al. Information provision for stroke patients and their caregivers. Cochrane Database Syst Rev 2012; 11: CD001919.

73. Brady MC, Kelly H, Godwin J, et al. Speech and language therapy for aphasia following stroke. Cochrane Database Syst Rev 2016; 6: CD000425.

74. Legg LA, Lewis SR, Schofield-Robinson OJ, et al. Occupational therapy for adults with problems in activities of daily living after stroke. Cochrane Database Syst Rev 2017; 7: CD003585.

75. Saunders DH, Sanderson M, Hayes S, et al. Physical fitness training for stroke patients. Cochrane Database Syst Rev 2016; 3: CD003316.

76. English C, Hillier SL and Lynch EA. Circuit class therapy for improving mobility after stroke. Cochrane Database Syst Rev 2017; 6: CD007513.

77. Vienneau D, de Hoogh K, Faeh D, et al. More than clean air and tranquillity: residential green is independently associated with decreasing mortality. Environ Int 2017; 108: 176-184.

78. de Gelder R, Koster EM, van Buren LP, et al. Differences in adults' health and health behaviour between 16 European urban areas and the associations with socio-economic status and physical and social environment. Eur J Public Health 2017; 27: 93-99.

79. Aziz NA, Leonardi-Bee J, Phillips M, et al. Therapybased rehabilitation services for patients living at home more than one year after stroke. Cochrane Database Syst Rev 2008; 2: CD005952.

80. Pollock A, Baer G, Campbell P, et al. Physical rehabilitation approaches for the recovery of function and mobility following stroke. Cochrane Database Syst Rev 2014; 4: CD001920.

81. Mehrholz J, Thomas S and Elsner B. Treadmill training and body weight support for walking after stroke. Cochrane Database Syst Rev 2017; 8: CD002840.

82. Mehrholz J, Pohl M, Platz T, et al. Electromechanical and robot-assisted arm training for improving activities of daily living, arm function, and arm muscle strength after stroke. Cochrane Database Syst Rev 2015; 11: CD006876.

83. Morris R. Meeting the psychological needs of community-living stroke patients and carers: a study of third sector provision. Disabil Rehabil 2016; 38: $52-61$.

84. Turner-Stokes L, Pick A, Nair A, et al. Multi-disciplinary rehabilitation for acquired brain injury in adults of working age. Cochrane Database Syst Rev 2015; 12: CD004170.

85. European Stroke Oranisation. Guidelines directory, https://eso-stroke.org/eso-guideline-directory (accessed 1 May 2018).

86. Norrving B, Bray BD, Asplund K, et al. Cross-national key performance measures of the quality of acute stroke care in Western Europe. Stroke 2015; 46: 2891-2895.

87. Aziz NA, Pindus DM, Mullis R, et al. Understanding stroke survivors' and informal carers' experiences of and need for primary care and community health services $-\mathrm{a}$ systematic review of the qualitative literature: protocol. BMJ Open 2016; 6: e009244.

88. Sacco RL, Sandercock P, Endres M, et al. Review and prioritization of stroke research recommendations to address the mission of the World Stroke Organization: a call to action from the WSO Research Committee. Int J Stroke 2015; 10: 4-9.

89. World Stroke Organisation. Global Stroke Bill of Rights, www.worldstrokecampaign.org/images/global_ stroke_bill_of_rights/English_GlobalBORights_web. pdf (accessed 11 October 2018).

90. McKevitt C, Fudge N, Redfern J, et al. Self-reported long-term needs after stroke. Stroke 2011; 42: 1398-1403.

91. Murray J, Young J, Forster A, et al. Feasibility study of a primary care-based model for stroke aftercare. $\mathrm{Br} J$ Gen Pract 2006; 56: 775-780.

92. Daniel K, Wolfe CD, Busch MA, et al. What are the social consequences of stroke for working-aged adults? A systematic review. Stroke 2009; 40: e431-e440.

93. Stroke Association. Feeling overwhelmed: the emotional impact of stroke, www.stroke.org.uk/sites/default/ files/feeling_overwhelmed_final_web_0.pdf (accessed 11 October 2018).

94. Department of Health. National Stroke Strategy, December 2007. http://webarchive.nationalarchives.gov. uk/20130105121530/http://www.dh.gov.uk/en/ Publicationsandstatistics/Publications/Publications Policyandguidance/dh_081062 (accessed 11 October 2018).

95. Pindus DM, Mullis $\bar{R}$, Lim L, et al. Stroke survivors' and informal caregivers' experiences of primary care and community healthcare services - a systematic review and meta-ethnography. PLoS One 2018; 13: e0192533.

96. Katzan IL, Thompson NR, Lapin B, et al. Added value of patient-reported outcome measures in stroke clinical practice. J Am Heart Assoc 2017; 6: e005356.

97. Riksstroke Brief summary of data for the full year 2016, www.riksstroke.org/wp-content/uploads/2017/11/Briefsummary-of-Riksstroke-data-for-the-full-year-2016.pdf (accessed 11 October 2018).

98. Nilsson E, Orwelius L and Kristenson M. Patientreported outcomes in the Swedish National Quality Registers. J Intern Med 2016; 279: 141-153.

99. Demuth HU, Dijkhuizen RM, Farr TD, et al. Recent progress in translational research on neurovascular and neurodegenerative disorders. Restor Neurol Neurosci 2017; 35: 87-103.

100. Bosetti F, Koenig JI, Ayata C, et al. Translational stroke research: vision and opportunities. Stroke 2017; 48: 2632-2637.

101. Smart N, Pries AR and Dirnagl U. Dr Smart talks to Prof Pries and Prof Dirnagl on animal experimentation in biomedical research. Cardiovasc Res 2017; 113: e12-e15.

102. Neuhaus AA, Couch Y, Hadley G, et al. Neuroprotection in stroke: the importance of collaboration and reproducibility. Brain 2017; 140: 2079-2092.

103. De Meyer SF, Denorme F, Langhauser F, et al. Thromboinflammation in stroke brain damage. Stroke 2016; 47: 1165-1172. 
104. Savitz SI, Baron JC, Yenari MA, et al. Reconsidering neuroprotection in the reperfusion era. Stroke 2017; 48: 3413-3419.

105. Anrather $\mathbf{J}$ and Iadecola C. Inflammation and stroke: an overview. Neurotherapeutics 2016; 13: 661-670.

106. Walker KA, Power MC, Hoogeveen RC, et al. Midlife systemic inflammation, late-life white matter integrity, and cerebral small vessel disease: the Atherosclerosis Risk in Communities Study. Stroke 2017; 48: 3196-3202.

107. Yu IC, Kuo PC, Yen JH, et al. A combination of three repurposed drugs administered at reperfusion as a promising therapy for postischemic brain injury. Transl Stroke Res 2017; 8: 560-577.

108. Zhou Y, Wang Y, Wang J, et al. Inflammation in intracerebral hemorrhage: from mechanisms to clinical translation. Prog Neurobiol 2014; 115: 25-44.

109. Tan R, Traylor M, Rutten-Jacobs L, et al. New insights into mechanisms of small vessel disease stroke from genetics. Clin Sci (Lond) 2017; 131: 515-531.

110. Haffner C, Malik R and Dichgans M. Genetic factors in cerebral small vessel disease and their impact on stroke and dementia. J Cereb Blood Flow Metab 2016; 36: $158-171$.

\section{Appendix I. Members of the working group}

The Steering Committee consisted of Bo Norrving (chair; Lund, Sweden), Valeria Caso (co-chair; Perugia, Italy), Martin Dichgans (Munich, Germany), Joanna M Wardlaw (Edinburgh, UK), Charlotte Cordonnier (Lille, France), Robert Mikulik (Brno, Czech Republic), Alla Guekht (Moscow, Russia), Antonio Davalos (Girona, Spain), Kursad Kutluk (Izmir, Turkey), and Jon Barrick (London, UK).

Members of the ESAP working groups were as follows. Primary prevention: Edo Richard (Chair), Department of Neurology, Donders Institute for Brain, Cognition and Behaviour, Radboud University Medical Centre, Nijmegen, and Department of Neurology, Academic Medical Centre, Amsterdam, the Netherlands; Eivind Berge, Departments of Internal Medicine and Cardiology, Oslo University Hospital, Oslo, Norway; Catarina Fonseca, Hospital de Santa Maria, University of Lisbon, Lisbon, Portugal; Richard Hobbs, Nuffield Department of Primary Care Health Sciences, University of Oxford, Oxford, UK; Stefan Kiechl, Department of Neurology, Medical University of Innsbruck, Innsbruck, Austria; Patrik Michel, Stroke Center, Neurology Service, Lausanne University Hospital, Lausanne, Switzerland; Jelena Misita, Stroke Alliance For Europe, Brussels, Belgium; Violetta Mkrtchyan, Research and Clinical Center for Neuropsychiatry of Moscow, Moscow, Russia; Peter Rothwell, ${ }^{*}$ Centre for Prevention of Stroke and Dementia, University of
Oxford, Oxford, UK; and Jakko Tuomilehto, Dasman Diabetes Institute, Dasman, Kuwait, and National Institute for Health and Welfare, Helsinki, Finland. Organization of stroke services: Darius Nabavi (Chair), Department of Neurology, Vivantes Hospital Neukölln, Berlin, Germany; Hanne Christensen," Bispebjerg Hospital and University of Copenhagen, Copenhagen, Denmark; Miquel Gallofré, Stroke Program of Catalonia, Barcelona, Spain; Christian Gerloff, Department of Neurology, University Medical Center Hamburg Eppendorf (UKE), Hamburg, Germany; Damian Jenkinson, Dorset County Hospital NHS Foundation Trust, Dorchester, UK; Aleksandra Pavlovic, Faculty of Medicine, University of Belgrade, Belgrade, Serbia; Thorsten Steiner, Department of Neurology, Klinikum Frankfurt Höchst, Frankfurt, and Department of Neurology, Heidelberg University Hospital, Heidelberg, Germany; Danilo Toni, Department of Human Neurosciences, University La Sapienza, Rome, Italy; Alexander Tsiskaridze, Faculty of Medicine Tbilisi State University, Tbilisi, Georgia; and Georgios Tsivgoulis, Second Department of Neurology, Attikon University Hospital, National and Kapodistrian University of Athens, Athens, Greece. Management of acute stroke: Carlos A Molina (Chair), Department of Neurology, Hospital Vall d'Hebron Barcelona, Barcelona, Spain; Anita Arsovska, University Clinic of Neurology, University Ss Cyril and Methodius, Skopje, Macedonia; Heinrich Audebert, Center for Stroke Research Berlin, Charité Universitätsmedizin Berlin, Berlin, Germany; Urs Fischer, Department of Neurology, University Hospital Bern, Bern, Switzerland; Zuzana Gdovinova, Department of Neurology, Faculty of Medicine, PJ Šafárik University and University Hospital L Pasteur, Kosice, Slovakia; Werner Hacke, ${ }^{*}$ University of Heidelberg, Heidelberg, Germany; Catharina JM (Karin) Klijn, ${ }^{*}$ Department of Neurology, Donders Institute for Brain, Cognition and Behaviour, Radboud University Medical Center, Nijmegen, the Netherlands; Kennedy $\mathrm{R}$ Lees, Institute of Cardiovascular and Medical Sciences, University of Glasgow, Glasgow, UK; Mikael Mazighi, Lariboisière Hospital and Foundation Rothschild Hospital, Paris, France; and Bart van der Worp, Department of Neurology and Neurosurgery, University Medical Center Utrecht, Utrecht, the Netherlands. Secondary prevention and follow-up: Philip Bath (Chair), Stroke Trials Unit, Division of Clinical Neuroscience, University of Nottingham, Nottingham, UK; Zauresh Akhmetzhanova, National Centre for Neurosurgery, Astana, Republic of Kazakhstan; Hugues Chabriat, Department of 
Neurology, Hopital Lariboisiére, Université Denis Diderot, Paris, France; Frank-Eric de Leeuw, Department of Neurology, Donders Institute for Brain, Cognition and Behaviour, Radboud University Nijmegen Medical Center, Nijmegen, the Netherlands; Hans-Christoph Diener, Department of Neurology, University Hospital Essen, Essen, Germany; JeanLouis Mas, " Department of Neurology, Hôpital Sainte-Anne, Université Paris Descartes, Paris, France; Ivan Milojevic, Department of Neurology, Cuprija, Serbia; Serefnur Ozturk, Faculty of Medicine, Selcuk University, Konya, Turkey; Maurizio Paciaroni, ${ }^{*}$ Stroke Unit and Division of Cardiovascular Medicine, Santa Maria della Misericordia Hospital, University of Perugia, Perugia, Italy; and Alastair Webb, Centre for Prevention of Stroke and Dementia, University of Oxford, Oxford, UK. Rehabilitation: Katharina S Sunnerhagen (Chair), Institute of Neuroscience and Physiology, Sahlgrenska University Hospital, Gothenburg, Sweden; Michael Brainin, Danube University, Krems, Austria; Jesse Dawson," Institute of Cardiovascular and Medical Sciences, College of Medical, Veterinary and Life Sciences, University of Glasgow, Glasgow, UK; Peter Langhorne, Institute of Cardiovascular and Medical Sciences, University of Glasgow, Glasgow, UK; Didier Leys, Inserm U1171, Univ-Lille, CHU Lille, Hôpital Roger Salengro, Lille, France; Monique Lindhout, Stroke Alliance for Europe/Dutch Brain Injury Association (Hersenletsel.nl), Velp, the Netherlands; Andreas Luft, University Hospital Zurich and Cereneo Center for Neurology and Rehabilitation, Vitznau, Switzerland; Gillian Mead, University of Edinburgh, Edinburgh, UK; Jan Mehrholz, Technical University Dresden, Faculty of Medicine, Dresden, Germany; and Marion Walker, Faculty of Medicine and Health Sciences, University of Nottingham, Nottingham, UK. Evaluation of outcomes and quality: Anthony Rudd (Chair), Guy's and St Thomas' NHS Foundation Trust and National Clinical Director for Stroke NHS England, London, UK; Sonia Abilleira, Stroke Programme/Agency for Health Quality and Assessment of Catalonia, Barcelona, Spain; Yannick Béjot, Dijon Stroke Registry, University Hospital and Medical School of Dijon, University of Burgundy, Dijon, France; André de Rosa Spierings, Stroke Alliance For Europe (SAFE)/Hersenletsel.nl, Velp, the Netherlands; Marie Eriksson," Department of Statistics, USBE, Umeå University, Sweden; Joseph Harbison, Department of Medical Gerontology, University of Dublin, Trinity College, Dublin, Ireland; Tatiana Kharitonova, Saint Petersburg Research Institute of Emergency Medicine, St Petersburg, Russia; Dilek Necioğlu Orken, Department of Neurology, Istanbul Bilim University,
Istanbul, Turkey; Francesca Pezella, San CamilloForlanini Hospital, Rome, Italy; and Igor Voznuk, Saint Petersburg Research Institute of Emergency Medicine, St Petersburg, Russia. Translational research priorities: Anna Planas (Chair), Institut d'Investigacions Biomèdiques de Barcelona (IIBB), Consejo Superior de Investigaciones Científicas (CSIC), Institut d'Investigacions Biomèdiques August Pi i Sunyer (IDIBAPS), Barcelona, Spain; Denis Vivien, Université Normandie, CHU de Caen Normandie, Caen, France; Stephanie Debette, Bordeaux Population Health Research Center and Department of Neurology, CHU de Bordeaux, Bordeaux, France; Adam Denes, Institute of Experimental Medicine, Hungarian Academy of Sciences, Budapest, Hungary; Ulrich Dirnagl, Charite Universitätsmedizin Berlin and Berlin Institute of Health, Berlin, Germany; Arthur Liesz, Institute for Stroke and Dementia Research, Klinikum der Universität München, Munich, Germany; Hugh Stephan Markus, Clinical Neuroscience, University of Cambridge, Cambridge, UK; and Geert Jan Biessels, Department of Neurology, University Medical Center Utrecht, Utrecht, the Netherlands . Life after stroke: Avril Drummond (Chair), School of Health Sciences, University of Nottingham, Nottingham, UK; Juliet Bouverie, Stroke Association, London, UK; Anne Gordon, Guy's \& St Thomas' Hospital NHS Foundation Trust and King's College London, London, UK; Janika Kõrv, ${ }^{*}$ Department of Neurology and Neurosurgery, University of Tartu, Tartu, Estonia; Charlotte Magnusson, Department of Design Sciences, Lund University, Lund, Sweden; Chris McKevitt, 'School of Population Health \& Environmental Sciences, King's College, London, UK; Harriet Proios, Department of Educational and Social Policy, University of Macedonia, Thessaloniki, Greece; Markus Wagner, Stroke Alliance For Europe, Brussels, Belgium; and Diana Wong, SAFE-Stroke Alliance for Europe, Lisbon, Portugal.

*Members responsible for defining research priorities.

\section{Appendix 2. Issues in secondary prevention of stroke}

Accurate data are scarce, with few countries having national registries and most published data being 5-10 years old. Hence, there is an urgent need for contemporaneous accurate monitoring and reporting. In an ESO survey of national stroke societies performed in 2017 , there was wide variation in the availability of evidence-based secondary prevention treatments: in most Western European countries, TIAs were routinely 
assessed on the same day or within $48 \mathrm{~h}$, usually as an inpatient or in a dedicated TIA clinic, whereas in some Eastern European countries TIA patients were still routinely reviewed in general medical clinics or primary care, often after more than a week. Although most patients undergo carotid imaging by ultrasound and 24-48-h monitoring for AF, few countries offer more prolonged monitoring to the majority of patients, and assessment by ECG alone remains the commonest method in some Eastern European countries. Across Europe, blood pressure after stroke or TIA is most often assessed by clinic readings and ongoing management is commonly devolved to primary care; few countries regularly use ambulatory or home monitoring of blood pressure.

Less than half of countries routinely provide lifestyle management programmes after stroke, although such programmes are more common in Western Europe; only smoking cessation programmes are consistently available, and reimbursement for such programmes is rare. Adherence to antiplatelet therapy is high, but after one year, fewer than half of patients are estimated to be still on statins in some countries. While $60-80 \%$ of patients in the highest performing countries report blood pressure control at one year, significantly lower national rates of control are common. Interventional carotid endarterectomy is widely available, but the time to treatment is highly variable, from within a week for patients in the best-performing nations to routine waits of greater than one month in others. Finally, although advanced procedures (carotid stenting, atrial appendage/PFO closure, neurosurgery and radiosurgery) were more commonly available overall in the west, their use varied substantially, largely independently of the economic background of the reporting nation.

Overall, provision of secondary prevention services is highly variable in Europe, with a consistent east-west divide for many interventions. Improving the availability of lifestyle management, rates of risk factor control and treatment adherence, and increased access to interventions, is needed across Europe. 